\title{
Organization and engagement of a prefrontal-olfactory network during olfactory selective attention
}

Hillary L. Cansler', Estelle E. in 't Zandt', Kaitlin S. Carlson'1, Waseh T. Khan ${ }^{1}$, Minghong $\mathrm{Ma}^{2}$, Daniel W. Wesson ${ }^{1}$

${ }^{1}$ Department of Pharmacology and Therapeutics

Center for Smell and Taste

Center for Addiction Research and Education

Norman Fixel Institute for Neurological Diseases

University of Florida

1200 Newell Dr.

Gainesville, FL 32610, USA

2Department of Neuroscience, University of Pennsylvania Perelman School of Medicine, Philadelphia, PA 19104, USA

Contact:

hillary.cansler@ufl.edu

Acknowledgements: This work was supported by National Institutes of Health grants R01DC016519 and R01DC014443 to DW, R01DA049545, R01DA049449, and R01 NS117061 to MM and DW, R01DC006213 to MM, and F32DC018232 to HC. We thank Dr. Mark Fuccillo for generously sharing reagents.

Conflicts of Interest: The authors have no perceived or real conflicts of interest to declare.

Key words: sniffing; olfactory cortex; prefrontal cortex; neural oscillations; active sampling; neural circuits. 


\section{Abstract}

2 Sensory perception is profoundly shaped by attention. Attending to an odor strongly

3 regulates if and how a smell is perceived - yet the brain systems involved in this process

4 are unknown. Here we report integration of the medial prefrontal cortex (mPFC), a

5 collection of brain regions integral to attention, with the olfactory system in the context of

6 selective attention to odors. First, we used tracing methods to establish the tubular

7 striatum (TuS, also known as the olfactory tubercle) as the primary olfactory region to

8 receive direct mPFC input in rats. Next, we recorded local field potentials from the

9 olfactory bulb (OB), mPFC, and TuS while rats completed an olfactory selective attention

10 task. Gamma power and coupling of gamma oscillations with theta phase were

11 consistently high as rats flexibly switched their attention to odors. Beta and theta

12 synchrony between mPFC and olfactory regions were elevated as rats switched their

13 attention to odors. Finally, we found that sniffing was consistent despite shifting

14 attentional demands, suggesting that the mPFC-OB theta coherence is independent of

15 changes in active sampling. Together, these findings begin to define an olfactory attention

16 network wherein mPFC activity, as well as that within olfactory regions, are coordinated

17 in manners based upon attentional states. 


\section{Introduction}

19 Sensory processing and thus perception are both profoundly shaped by our everchanging cognitive states. In most cases, the thalamus appears to be a major driver of

21 state-dependent modulation of sensory information (Wimmer et al. 2015; O'Connor et al.

22 2002; Halassa and Kastner 2017; McCormick and Feeser 1990). For instance, the visual

23 thalamus modulates primary visual cortex in manners that enhances the signal to noise

24 of an attended visual cue (McAlonan et al. 2008). Similarly, the gustatory thalamus

25 regulates taste-evoked responsivity of gustatory cortex neurons (Samuelsen et al. 2013).

26 Among all sensory systems, the olfactory system presents a unique challenge for

27 understanding the influence of cognitive state upon sensory processing. This is because,

28 while humans (Zelano et al. 2005; Spence et al. 2000; Plailly et al. 2008) and rodents

29 (Carlson et al. 2018) alike can selectively attend to odors, the organization of the olfactory

30 system lacks obligatory thalamic processing (Gottfried 2010; Courtiol and Wilson 2016;

31 Kay and Sherman 2006). Thus, other brain systems must engage with olfactory

32 processing in order to afford one the ability to attend to odors. This is a significant issue

33 since odors are most often encountered in highly multisensory environments, for instance

34 during eating, wherein potentially distracting or conflicting cues must be ignored at the

35 expense of selectively attending to odor.

Truly very little is known regarding the neural mechanisms underlying olfactory

37 attention. One brain region that seems likely to confer this ability, at least in part, is the 38 tubular striatum (TuS, also known as the olfactory tubercle (Wesson 2020)). This is true

39 in both humans and rodents. For instance, early work using fMRI uncovered the first 40 evidence that the human TuS is more activated in response to attended versus 
41 unattended odors while human subjects engaged in an olfactory selective attention task

42 (Zelano et al. 2005). Importantly, attention-dependent amplification of odor-evoked

43 activity in the TuS exceeded that of even the primary "piriform" olfactory cortex (PCX)

44 (Zelano et al. 2005). Our group subsequently found that odor-evoked signal to noise

45 among TuS neurons was enhanced as rats engaged in olfactory selective attention.

46 (Carlson et al. 2018). While the TuS is engaged by attention in manners which may

47 subserve the ability to attend to odors, the way that the TuS integrates into a wider brain

48 network in the context of odor-directed attention is unknown. This includes major voids in

49 our understanding of descending inputs from brain regions known to be integral for

50 attention, and how TuS activity is structured relative to that of these regions.

51 The rodent prefrontal cortex (PFC), depending upon how one chooses to define it

52 (Laubach et al. 2018; Le Merre et al. 2021), comprises several key subregions including

53 but not limited to the medial PFC (mPFC) and the orbitofrontal cortex (OFC), each of

54 which can be divided into more specific subregions. The medial prefrontal cortex (mPFC)

55 is crucial for many executive processes including attention (Wimmer et al. 2015; Birrell

56 and Brown 2000; Kim et al. 2016; Miller and Cohen 2001) and is highly interconnected

57 with the rest of the brain (Le Merre et al. 2021), with particularly dense inputs to sensory

58 and thalamic areas. mPFC neurons are modulated as animals attentively await a stimulus

59 (Rodgers and DeWeese 2014; Kim et al. 2016), and disruptions to the mPFC impair

60 attentional set-shifting (Birrell and Brown 2000; Ragozzino et al. 2003), sustained

61 attention (Kim et al. 2016), and selective attention (Wimmer et al. 2015). mPFC

62 subdivisions include the prelimbic (PrL) and infralimbic (IL) cortices, which seem to

63 possess dissociable behavioral functions (Hardung et al. 2017; Marquis et al. 2007; de 
64 Kloet et al. 2021; Luchicchi et al. 2016). Specifically, the PrL appears important for set-

65 shifting and selective attention (Marquis et al. 2007; Schmitt et al. 2017; Kim et al. 2016;

66 Rodgers and DeWeese 2014), while the IL is implicated in behavioral flexibility and

67 extinction (Barker et al. 2014). Therefore, the mPFC, especially the PrL and IL, are

68 putative candidates for influencing olfactory processing via top-down modulation during

69 attentional states.

Local field potential (LFP) oscillations in the gamma band $(40-100 \mathrm{~Hz})$ in sensory

71 and prefrontal cortices have been associated with attention (Fries et al. 2001; Vinck et al.

72 2013; Borgers et al. 2005; Brassai et al. 2015; Schroeder and Lakatos 2009a). In the

73 olfactory system, increased power of gamma oscillations in the olfactory bulb (OB) is

74 observed during successful discriminations between perceptually demanding odor pairs

75 (Beshel et al. 2007), and learning (Losacco et al. 2020a), suggesting that they in some

76 manner aid in cognitively demanding processes. In addition to high frequency oscillations,

77 theta oscillations $(2-12 \mathrm{~Hz})$ are profoundly shaped by respiration, including fast

78 investigatory sniffing, in olfactory regions and beyond (Adrian 1942; Macrides et al. 1982;

79 Vanderwolf 1992; Tort et al. 2018b; Colgin 2013; Zhang et al. 2021; Fontanini and Bower

80 2006; Kay and Laurent 1999; Buonviso et al. 2003; Miura et al. 2012). Interestingly, theta-

81 band coherence is elevated between the OB and mPFC in emotionally-salient contexts

82 (Moberly et al. 2018; Bagur et al. 2021; Zhong et al. 2017), indicating functional

83 connectivity between these networks that can be influenced by behavioral state.

84 Examining neural oscillations within individual structures, and their synchrony between

85 structures, can yield valuable insights into the ways that brain regions form functional

86 networks (Buzsaki 2006; Fries 2015). Whether the mPFC and olfactory system networks 
87 (either independently or together) are engaged by selective attention to odors has not

88 been explored.

Here, we sought to investigate the anatomical and functional integration of the

90 mPFC with olfactory regions (including the $\mathrm{OB}$ and TuS) in the context of selective

91 attention to odors. First, we used cell-type-specific tracing methods to reveal that

92 excitatory mPFC neurons preferentially target the TuS compared with other olfactory

93 regions. Next, we used multi-site LFP recordings to demonstrate that the OB, mPFC, and

94 TuS modify their activity in intra- and inter-areal manners during a behavioral task that

95 requires selective attention to odors and intermodal attentional switches. Interestingly,

96 through measuring sniffing as rats engaged in selective attention, we observed that rats

97 covertly display odor-directed attention, maintaining highly stereotyped sniffing structured

98 to the task despite shifting attentional demands. Together this work adds to our

99 understanding of the organization and activities of brain systems which are engaged

100 during olfactory attention.

101

102

\section{Results}

103 The PrL and IL preferentially target the TuS compared to other major olfactory structures.

104 The rodent PFC projects throughout the brain with notably strong and well characterized

105 inputs to the ventral striatum and thalamus (Vertes 2004; Le Merre et al. 2021). Yet the

106 connectivity of the PFC with primary $(\mathrm{OB})$ and secondary olfactory structures (the anterior

107 olfactory nucleus (AON), PCX, and TuS) is not well defined. To address this, we injected

108 Cre-dependent anterogradely transported AAVs encoding synaptophysin tagged with

109 either GFP or mRuby into the PrL or IL, in combination with an AAV encoding Cre under 
110 control of the CaMKII promotor (Fig. 1A-B) (Herman et al. 2016). This approach allowed

111 us to observe fluorescent puncta (which can more confidently be attributed to synaptic

112 terminals, rather than fibers of passage) in olfactory regions receiving input from either

113 the PrL or IL cortex. Importantly, because Cre expression was driven by the CaMKII

114 promotor, we can specifically assess excitatory projections which make up $>80 \%$ of

115 mPFC neurons (Erö et al. 2018) and are major regulators of the PFC's effects (de Kloet

116 et al. 2021). We quantified puncta in three structures recipient of dense olfactory bulb

117 input: the AON, PCX and TuS (Fig. 1B-G). We also inspected the OB for puncta, but did

118 not quantify this since none were detectable (Fig. 1F). Other than the OB, we observed

119 fluorescent puncta in all regions examined, with a striking density in the TuS. Specifically,

120 the medial division of the TuS (mTuS), which some have indicated plays a particularly

121 prominent role in olfaction and motivated behaviors (Ikemoto 2003; Murata et al. 2015;

122 Zhang et al. 2017), receives the most input from the mPFC, even more so than all other

123 regions combined (Fig. 1G). Within the mTuS, we observed synapses throughout all 3

124 layers, with significantly more in layers 2 and 3 (Fig. 1H). Importantly, this is where the

125 vast majority of medium spiny neurons, the principal neuron of the TuS, reside. Further,

126 we found that the IL projections to the mTuS are denser than those of the PrL (Fig. 1G-

127 H). Notably, in 2 separate rats we unilaterally injected a retrograde AAV encoding GFP

128 into the PrL and IL and observed no GFP+ cells in the TuS (or anterior PCX), indicating

129 that the there is no direct reciprocal feedback from the TuS (or anterior PCX) to the PrL

130 or IL (Fig. S1). Together, these findings indicate that the PrL and IL project to multiple

131 olfactory regions, with the mTuS being the primary recipient of this input. 

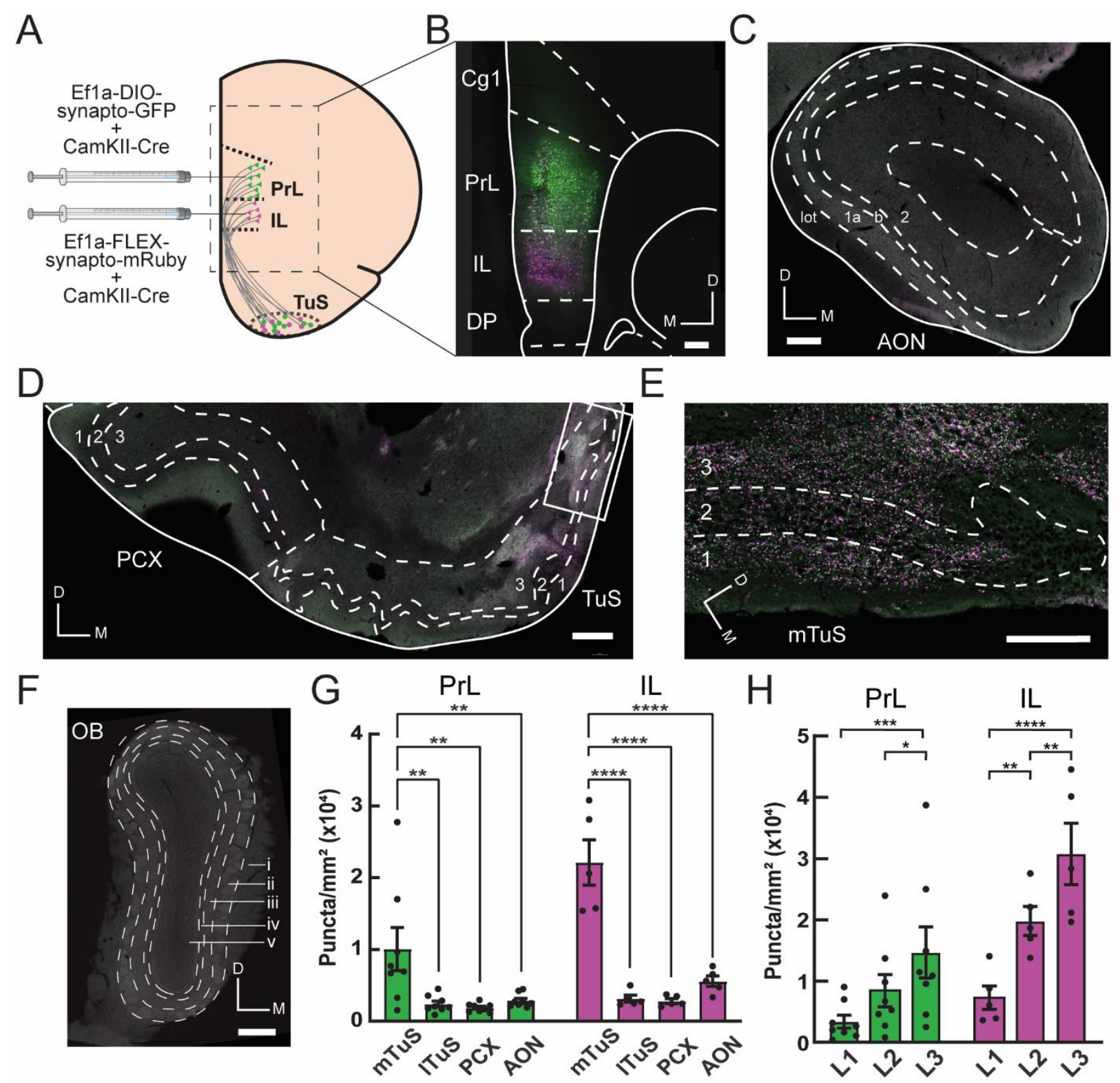

132

133

134

135

136

137

138

139

140

141

142

143

144

145

146

147

Figure 1. The prelimbic and infralimbic medial prefrontal cortex preferentially target the tubular striatum compared to other major olfactory structures. A. The PrL and IL cortices were selectively targeted with 50/50 mixtures of Ef1a-DIO-synaptophysin-GFP/pENN-AAV9CamKII-Cre-SV40 and Ef1a-FLEX-synaptophysin-mRuby/pENN-AAV9-CamKII-Cre-SV40, respectively. B. Representative $\mathrm{mPFC}$ image showing region-specific viral transduction within the same rat. Scale bar $250 \mu \mathrm{m}$. C. Representative image of the AON, showing few fluorescent puncta. Cell layers 1-2 and the lateral olfactory tract (lot) are indicated. Scale bar $250 \mu \mathrm{m}$. D. Representative image of the PCX and TuS. Note high fluorescence in the medial TuS and low fluorescence in the lateral TuS and PCX. Boxed region is shown in panel E. Scale bar $250 \mu \mathrm{m}$. E. Magnified view of boxed region shown in panel $D$, showing high levels of fluorescent puncta, indicating synaptic terminals from TuS-projecting mPFC neurons. This image has been digitally deconvolved to enhance clarity, for illustration purposes only. Scale bar $100 \mu \mathrm{m}$. F. Representative image of the OB absent of fluorescent puncta. Dashed lines indicate layers: i. olfactory nerve layer; ii. glomerular layer; iii. external plexiform layer; iv. mitral cell layer; v. granule cell layer. Scale bar $250 \mu \mathrm{m}$. G. Quantification of fluorescent puncta across olfactory regions, 
normalized by area of quantified region. PrL:one-way ANOVA, main effect of regions, $F(3,21)=7.82, p=0.001$. IL:one-way ANOVA, main effect of regions, $F(3,12)=37.08, p<0.0001$. Asterisks indicate results from Tukey's multiple comparisons test, ${ }^{* *} \mathrm{p}<0.01,{ }^{\star * \star *} \mathrm{p}<0.0001$. PrL mTuS vs. IL mTuS, unpaired t-test, $p=0.02$. H. Quantification of fluorescent puncta across layers in the mTuS. PrL: one-way ANOVA, main effect of layers, $F(2,14)=12.62, p=0.0007$. IL: one-way ANOVA, main effect of layers, $F(2,8)=43.04, p<0.0001$. Asterisks indicate results from Tukey's multiple comparisons test, ${ }^{*} \mathrm{p}<0.05,{ }^{* *} \mathrm{p}<0.01,{ }^{* * *} \mathrm{p}<0.001,{ }^{* * *} \mathrm{p}<0.0001$. PrL, prelimbic cortex; IL, infralimbic cortex; Cg1, cingulate area 1; DP, dorsal peduncular cortex; mTuS/ITuS, medial/lateral tubular striatum; PCX, piriform cortex; AON, anterior olfactory nucleus; OB, olfactory bulb; D, dorsal; $M$ medial; L1-L3, layer 1-3. PrL injection, $n=8$ rats; IL injection, $n=5$ rats. All error bars represent SEM.

Among PFC subregions, layer 5 PrL and IL neurons provide the densest input to the TuS.

While the PrL and IL are strongly implicated in attention, the PFC also includes the OFC.

The OFC is involved in polysensory processing (Rolls 2004; de Araujo et al. 2003; Small et al. 2001), and cognition and decision-making (for review see (Izquierdo 2017; Schoenbaum et al. 2009)), making it another strong candidate circuit to instruct statedependent odor processing. To directly compare the OFC $\rightarrow$ TuS and mPFC $\rightarrow$ TuS

166 pathways, we unilaterally injected a retrograde AAV encoding GFP into the TuS, and 167 quantified cell bodies throughout the PFC, including the PrL, IL, medial (MO), ventral 168 (VO), lateral (LO) and dorsolateral (DLO) OFC subdivisions (Fig. 2A-D). We found the 169 greatest numbers of cells in the IL, PrL, and $\mathrm{MO}$, which together make up the 170 ventromedial PFC (vmPFC) by virtue of their similar connectivity patterns (Le Merre et al.

171 2021). We observed significantly more cells in the IL than all other regions quantified

172 except the PrL, and significantly more cells in the PrL than all other areas regions 173 quantified except the IL and the MO (Fig. 2D). Thus, the PrL and IL provide the densest 174 inputs to the TuS and are well-positioned to influence odor processing.

175 Within the PrL and IL, we quantified cell body locations throughout the cortical 176 layers for both the ipsilateral and contralateral hemispheres. We observed that in both of 
177 these regions, the majority of cell bodies were found in layer 5 (Fig. 2E), which is 178 consistent with other glutamatergic (de Kloet et al. 2021) corticostriatal mPFC projections

179 (Nakayama et al. 2018; Ding et al. 2001; Gabbott et al. 2005). The fact that the TuS 180 receives its densest PFC inputs from the PrL and IL cortices, together with the fact that

181 these regions preferentially target the TuS over other olfactory regions, indicates that the

$182 \mathrm{mPFC} \rightarrow$ TuS pathway is likely the primary route whereby the olfactory system might 183 receive information regarding states or tasks requiring high executive function, including 184 attention. 
A

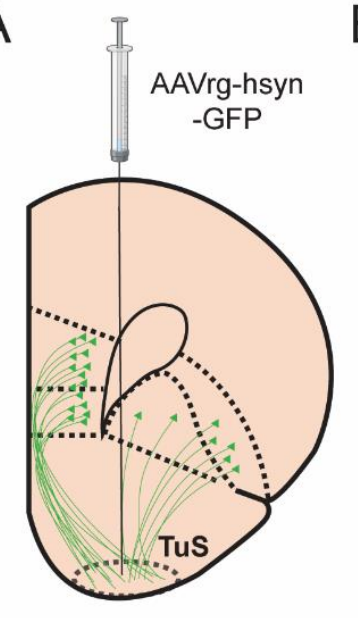

C

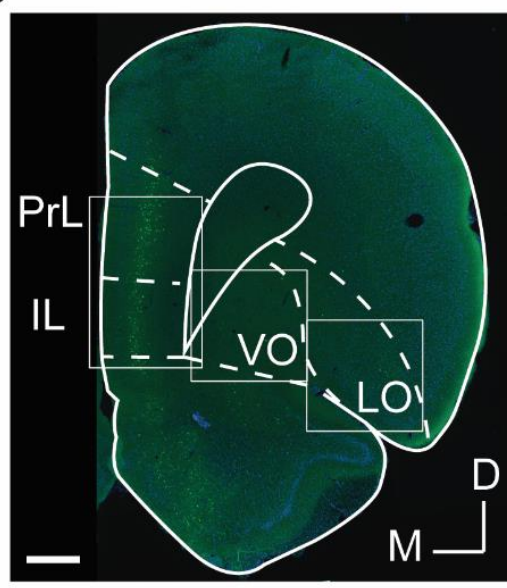

D

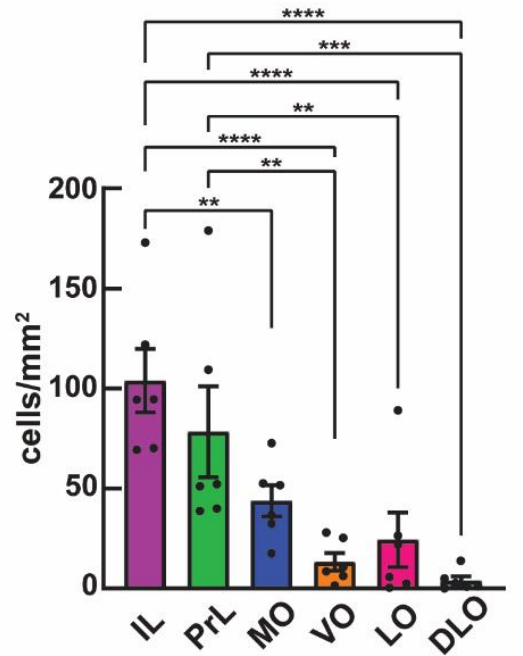

B

C
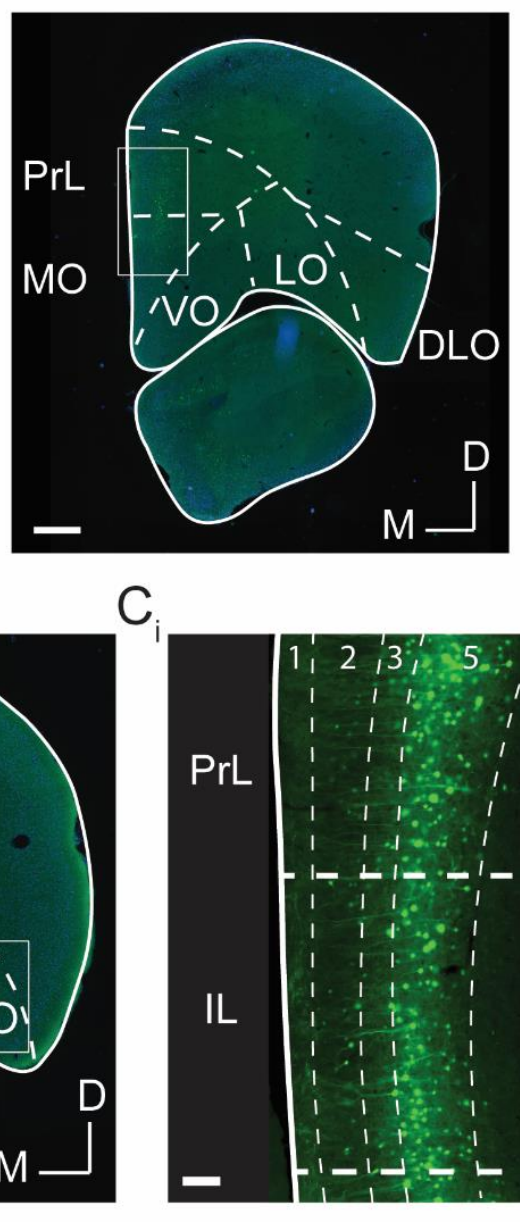

E
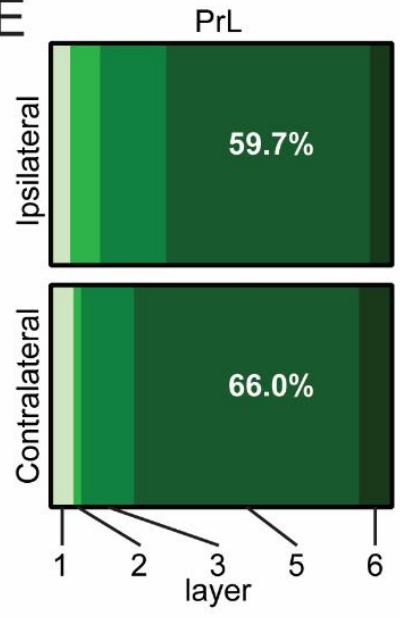

B

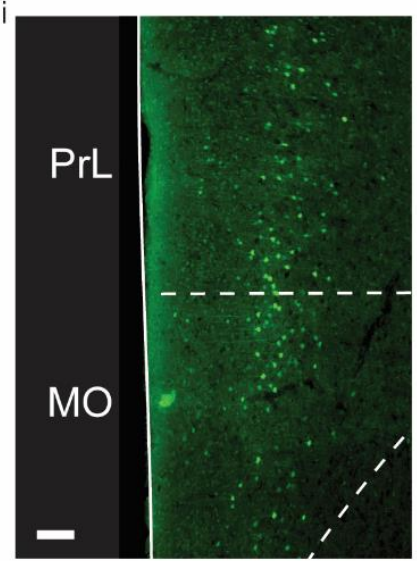

$\mathrm{C}_{\mathrm{ii}}$

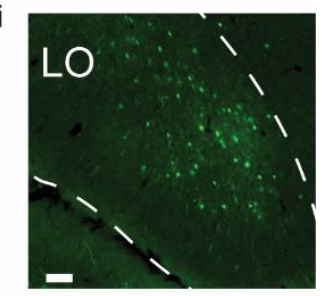

iii

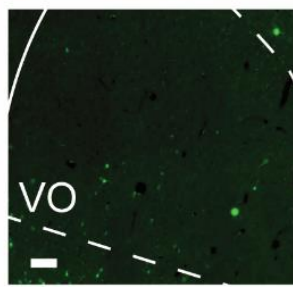

IL
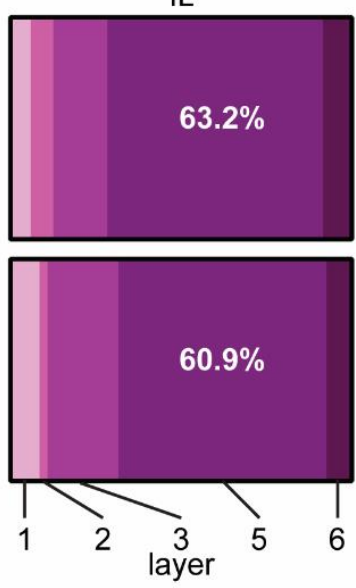

Figure 2. Among prefrontal cortex subregions, layer 5 prelimbic and infralimbic neurons provide the densest input to the tubular striatum. A. The TuS was injected with AAVrg-hsynGFP to identify TuS-projecting neurons throughout the prefrontal cortex. B. Representative mPFC image at Bregma $+4.2 \mathrm{~mm}$, showing GFP-labeled TuS-projecting neurons. Boxed region is indicated in panel $B_{i}$. Scale bar $500 \mu \mathrm{m}$. $B_{i}$. Magnified view of the boxed region in panel $B_{i}$. Scale bar $100 \mu \mathrm{m}$. C. Representative PFC image at Bregma $+3.2 \mathrm{~mm}$, showing GFP-labeled TuSprojecting neurons. Scale bar $500 \mu \mathrm{m}$. Ci. Magnified view of boxed region in panel $\mathrm{C}$ showing the 
PrL and IL cortices. Dotted lines indicate layers. Scale bar $100 \mu \mathrm{m}$. Cii. Magnified view boxed region showing LO cortex. Scale bar $100 \mu \mathrm{m}$. Ciii. Magnified view of boxed region showing VO cortex. Scale bar $100 \mu \mathrm{m} . \mathrm{n}=6$ rats. D. Quantification of cell numbers across prefrontal cortex regions ipsilateral to the injection site. One-way ANOVA, main effect of regions, $F(5,25)=15.67$, $p<0.0001$. Asterisks indicate results of Tukey's multiple comparisons test, ${ }^{* *} p<0.01$, ${ }^{* * *} p<0.001$, ${ }^{\star * * *} p<0.0001$. Error bars represent SEM. E. Distribution of cell bodies across PrL and IL layers, in both the contralateral and ipsilateral hemispheres, showing the majority of cell bodies are found in layer 5. PrL: Two-way ANOVA, main effect of layer $F(1.15,5.76)=11.48, p=0.014$. IL: Two-way ANOVA, main effect of layer $F(1.32,6.61)=42.55, p=0.0003$; main effect of hemisphere $F(1,5)=34.39, p=0.002$. $n=6$ rats.

Investigating mPFC and olfactory network activity during odor-directed selective attention.

We next sought to functionally test whether the mPFC and the olfactory system, specifically the $\mathrm{OB}$ and the TuS, integrate into a network during olfactory attention. To accomplish this, we combined multisite LFP recordings along with the Carlson Attention Task (CAT) (Carlson et al. 2018) to manipulate selective attention to odors (Fig. 3). Briefly, the CAT is a modified two-alternative choice task in which rats are simultaneously presented with one of two olfactory cues (odor A/odor B) and one of two auditory cues (tone on/tone off). A single behavioral session begins with tone attention: that is, the tone cues signal the reward port location whereas the odor cues are distractors (Fig. 3A-C, blue shading). Once criterion on the tone attention phase of the task has been reached (6 blocks of 20 trials at $\geq 80 \%$ correct), an uncued intermodal rule change occurs, and the rats must now direct their attention to odors, and ignore tones, to accurately locate their rewards (Fig. 3A-C, orange shading). This rule change is accompanied by a temporary drop in performance as the rats adjust their behavior to the new rule (Fig. 3C, pink shading) before they eventually perform well on odor attention ( 6 blocks at $\geq 80 \%$ correct, orange shading). We will refer to the blocks following the rule change before performance reaches $\geq 80 \%$ correct on odor attention as "switch" blocks, in which the rat is by-definition 
222 performing poorly. Importantly, each session began and ended with 3 blocks of odor-only

223 trials, in which there were no competing tone cues, to use as a control for odor

224 discrimination without any additional cognitive demand.

225 In the CAT, there are four possible combinations of trials (Fig. 3B). Two of these

226 are "congruent," in that the olfactory and auditory cues signal approach to the same

227 reward port, and two are incongruent, in that the cues signal opposite ports. Thus, the

228 correct reward port on incongruent trials depends on the current task rule: tone attention

229 (blue shading) or odor attention (orange shading). Importantly, all analyses of

230 physiological signals were limited to trials on which the tone was off to avoid multisensory

231 influences and focus on the effects of cognitive state on odor processing specifically

232 (Carlson et al. 2018). On a single trial of the CAT, the rat will nose poke to initiate a trial,

233 then must hold in the center port for 1 second before the stimuli come on (Fig. 3D, dark

234 gray shading). Then, the odor and tone stimuli come on simultaneously, and the rat must

235 remain in the center port for at least $400 \mathrm{~ms}$ sampling the stimuli (Fig. 3D, light gray

236 shading). After $400 \mathrm{~ms}$, the rat is free to make a choice at the left or right port and receive

237 a water reward if correct.

238 We simultaneously recorded LFPs from the TuS, mPFC, and OB from 5 highly-

239 proficient expert rats (see Methods) while they performed the CAT (Fig. 3D-E). This

240 allowed us to explore network dynamics locally within each structure, as well as coherent

241 activity between these structures. 
A

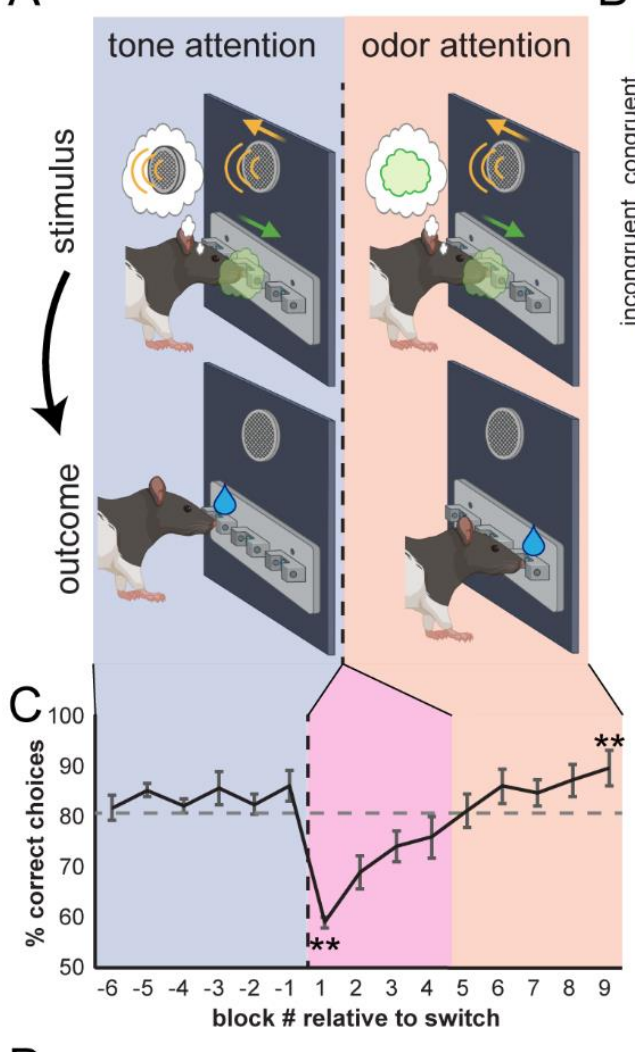

D

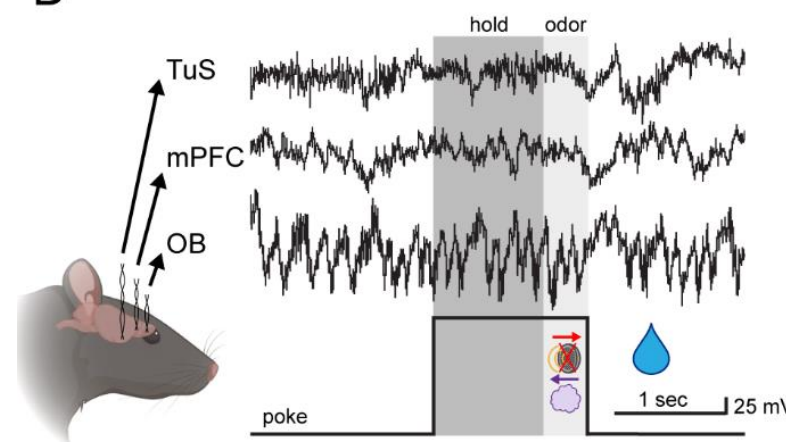

B

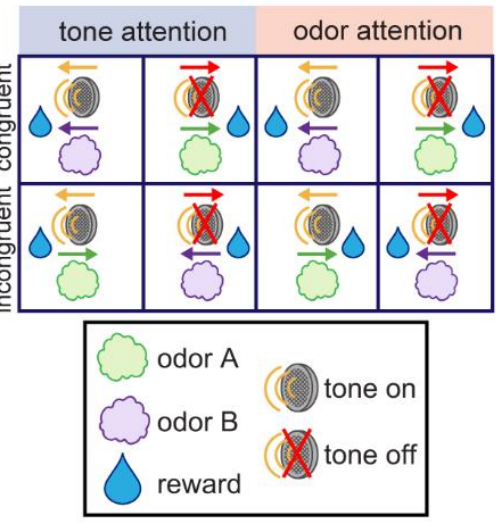

$\mathrm{E}$

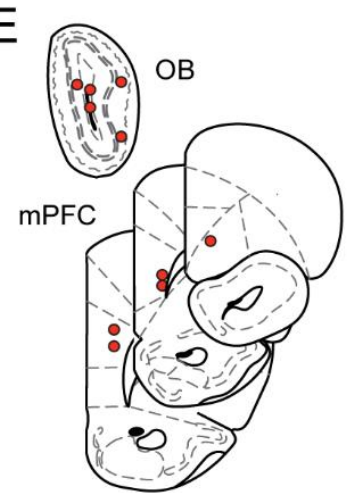

TuS

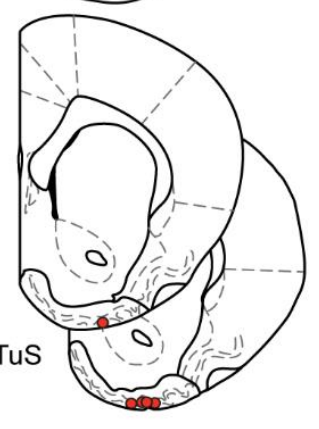

Figure 3. Investigating medial prefrontal cortex and olfactory network activity during odordirected selective attention. A. Freely-moving rats initiate a trial by nose-poking in a center port, which triggers simultaneous delivery of one of two auditory cues and one of two odors (stimulus). These cues direct the rat to retrieve a fluid reward at either the left or the right port (outcome). Behavioral sessions begin with tone attention (auditory cues predict reward; blue shading) and switch to odor attention (odors predict reward; orange shading). B. All possible trial combinations in the Carlson Attention Task. Half of these are congruent (odor and tone indicate same reward port) and half are incongruent (odor and tone indicate opposite reward ports). C. Behavioral performance of all rats across behavioral sessions. After completing 6 blocks of tone attention at criterion ( $\geq 80 \%$ correct; blue shading), the task was switched to odor attention (orange shading). Rats then switched their attention to odors and completed 6 blocks at criterion. Block -1 vs. 1 paired, two-tailed t-test, ${ }^{* *} \mathrm{p}=0.001$. Block 1 vs. 9 paired, two-tailed t-test, ${ }^{* *} \mathrm{p}=0.003 . \mathrm{n}=5$ rats, $4.6+/-0.5$ sessions per rat. Error bars represent SEM. D. All rats were implanted with bipolar recording electrodes in the OB, TuS, and mPFC, and LFPs were acquired during behavior. A sample trace is shown from a single trial, in which the rat pokes, holds in the center port for 1 
second awaiting stimuli (dark gray shading), and remains for $400 \mathrm{~ms}$ to sample the stimuli (light gray shading). E. Electrode location summary. Red dots indicate tips of bipolar LFP electrodes.

261 Elevations in gamma power upon intermodal switching and selective attention to odors.

262 Gamma oscillations are widely observed throughout the brain, and are tied to a diverse 263 array of functions including perception, memory, and attention (Fries et al. 2001; Buzsáki

264 and Wang 2012; Mably and Colgin 2018; Kim et al. 2016; Siegle et al. 2014; Cardin et al.

265 2009). Specifically, elevated gamma power in sensory neocortex is related to attentional

266 selection (Fries et al. 2001). In the OB, elevated gamma is linked to perceptually

267 demanding discriminations between perceptually similar odors (Beshel et al. 2007) and 268 has historically been conceptualized as integral to behavioral states (Martin and Ravel 269 2014; Eeckman and Freeman 1990). We examined gamma oscillations in the low (40-60 $270 \mathrm{~Hz})$ and high $(60-80 \mathrm{~Hz})$ gamma range within each brain structure as rats completed the

271 CAT (Fig 4). To do this, we measured the power of gamma oscillations within the hold

272 and odor trial epochs across each task type (odor only, tone attention, switch, and odor

273 attention), and normalized these values to those for odor only trials to highlight the specific

274 contributions of sensory-directed attention as compared to 'basic' olfactory discrimination.

275 In the TuS, we observed a slight enhancement in low gamma oscillations with

276 increased attentional demand, but this did not reach statistical significance across rats

277 (Fig. 4B). Interestingly, we observed that high gamma oscillations in the mPFC were

278 elevated during odor attention compared to tone attention (Fig. 4B). We were surprised

279 to observe this enhancement specifically for odor-directed attention in the mPFC, since

280 one might anticipate increased gamma power with increased cognitive demand

281 regardless of sensory modality. In the OB, we observed increased power of low gamma 282 oscillations during odor attention as compared to odor only, indicating that increased 
283 attentional demand alone is enough to modify odor information at the earliest stage of

284 processing in the brain (Fig. 4B). Finally, OB oscillations in the high gamma range were

285 elevated in power during the attentional switch relative to odor only and tone attention,

286 suggesting network activity related to cognitive flexibility. While we did uncover some

287 changes in beta band power (Fig. S2), these were not as dramatic across attentional

288 states as was the case with gamma. Overall, these findings indicate changes in local

289 network dynamics in the OB and the mPFC during selective attention to odors, suggesting

290 that attention may modulate odor processing at its most early processing stage (the OB).

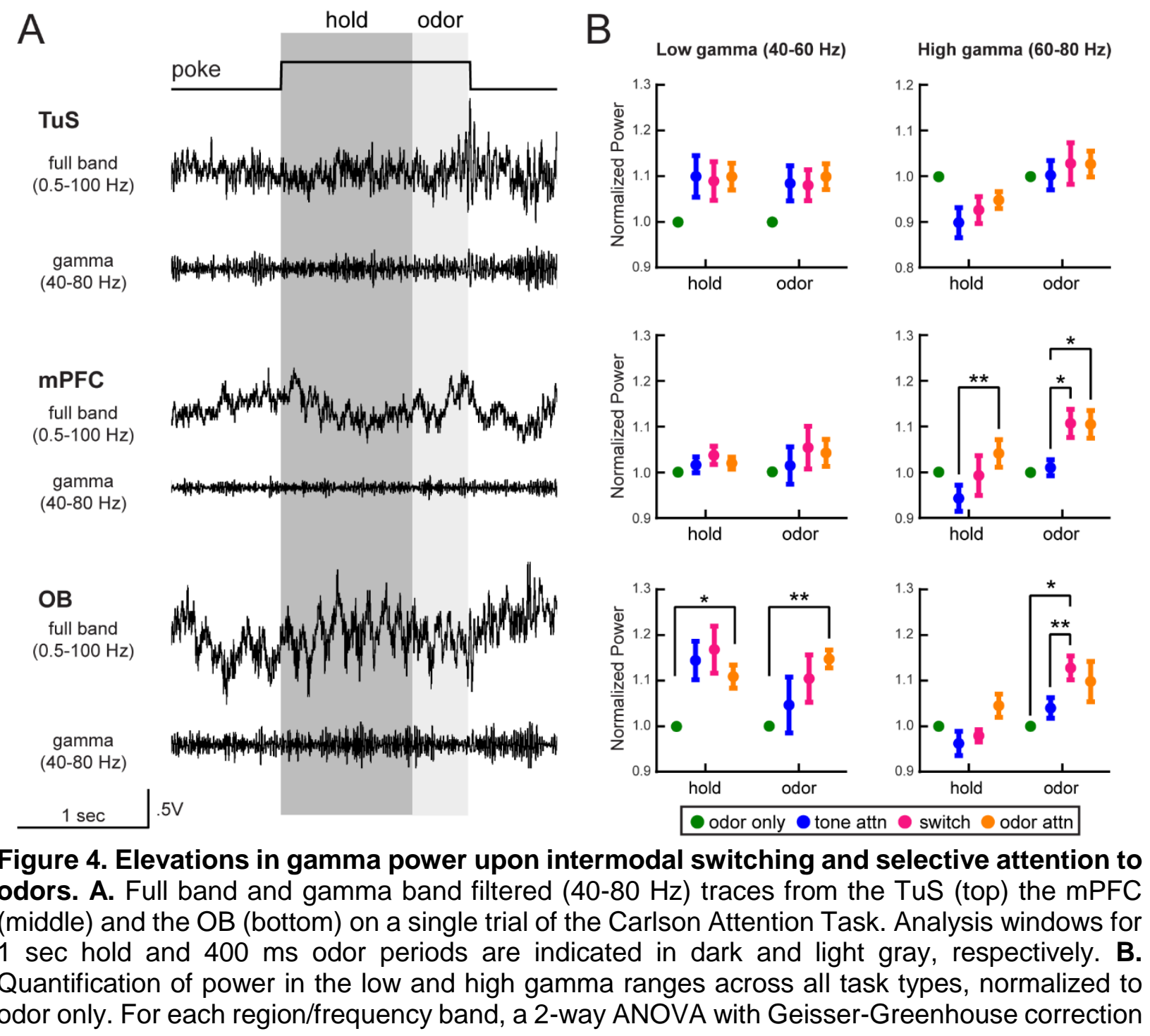


was completed. TuS, Low gamma: main effect of task type, $F(1.62,6.5)=6.04, p=0.037$. mPFC, high gamma: Main effect of trial epoch, $F(1.85,7.38)=13.66, p=0.004$. Interaction between trial epoch $x$ task type, $F(2.36,9.44)=5.91, p=0.019$. OB, high gamma: Main effect of trial epoch, $F(1.38,5.52)=9.47, p=0.02$. Main effect of task type, $F(2.22,8.88)=7.60, p=0.011 . n=5$ rats, 4.6 + +- 0.5 sessions per rat. On all graphs, asterisks indicate results from Tukey's multiple comparisons ${ }^{*} p<0.05,{ }^{* *} p<0.01$. All error bars represent SEM.

Olfactory bulb gamma oscillations couple with theta phase during selective attention.

307 In some brain regions, the amplitude of high frequency oscillations are structured by the phase of low frequency oscillations, a phenomenon known as phase amplitude coupling (PAC) (Tort et al. 2010; Bragin et al. 1995; Jensen and Colgin 2007; Lakatos et al. 2008; Canolty et al. 2006), which is considered a mechanism for attentional selection

311 (Schroeder and Lakatos 2009b). In the OB, PAC between high gamma and respiratory

312 theta becomes stabilized as mice become proficient at discriminating between odors

313 (Losacco et al. 2020a), indicating that learning and experience modulates OB PAC. This

314 plus our finding of elevated high gamma in the OB during odor directed attention led us

315 to investigate whether the OB network may engage in PAC during olfactory attention.

To address this, we first computed comodulograms to identify high frequency

317 oscillations coupled to theta phase within the rat OB during the CAT, which revealed

318 strong coupling between theta and high gamma (Fig 5A), and much weaker coupling

319 between theta and beta (Fig S3). To investigate the significance of theta-high gamma

320 PAC, we examined the trial-by-trial amplitude of high gamma power as a function of theta

321 phase, which indicated high coupling throughout individual sessions and across cognitive

322 states (Fig. 5B-C). Peak phase angle was consistent even comparing correct vs. incorrect

323 trials (Fig. 5D). For each task type, we computed the modulation index (MI) of the PAC,

324 which is a measure of the extent to which a given high-frequency oscillation is structured

325 to a low frequency carrier oscillation. MI values can range from $0.005-0.03$ from the 
326 hippocampus (Tort et al. 2010) and OB (Losacco et al. 2020b), and we observed a similar

327 range herein. The distribution in Fig. 5B shows the Ml theta-high gamma for an example

328 session, and indicates strong PAC. While some individual rats showed modulation of the

329 Ml with cognitive state, across the population there were no systematic changes in theta-

330 high gamma PAC with different attentional states (Fig. 5E). Because decreased variance

331 in the peak phase angle in the $\mathrm{OB}$ is associated with olfactory learning in a go no-go task

332 (Losacco et al. 2020a), we quantified this as well, but did not observe any differences

333 across cognitive states (Fig. 5E). Thus, while the tightly structured theta-high gamma

334 PAC in the $\mathrm{OB}$ does not change in magnitude across attentional states, its stability

335 suggests that it possibly supports expert, flexible cognitive function. 

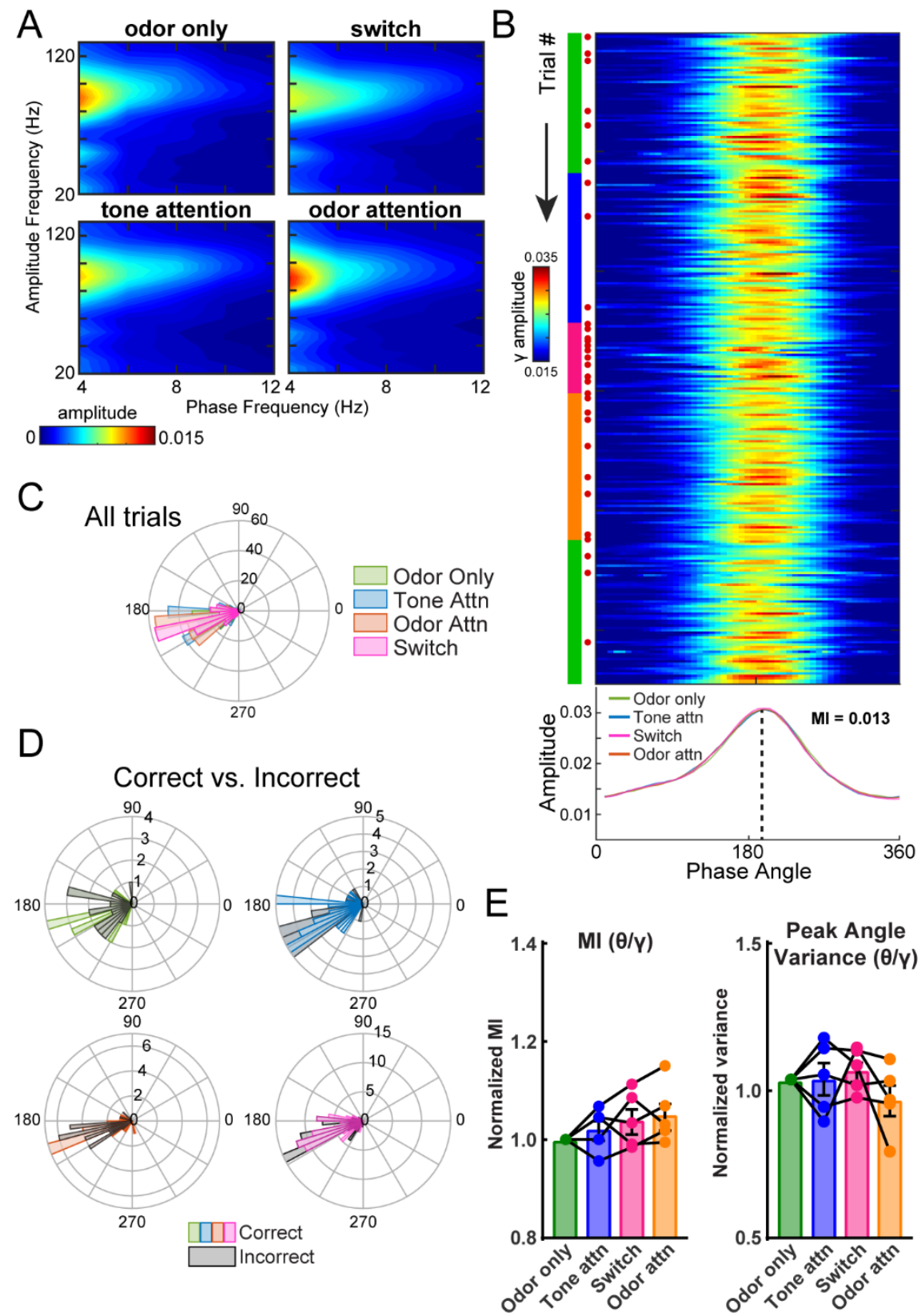

Figure 5. Olfactory bulb gamma oscillations couple with theta phase during selective attention. A. Mean comodulograms across rats showing strong coupling between high gamma and respiratory theta frequencies. $n=5$ rats, $4.6+/-0.5$ sessions per rat. B. Trial by trial thetagamma for one example session. Green, blue, pink, and orange markings on left side indicate current task type (odor only, tone attention, switch, and odor attention, respectively). Red dots indicate incorrect trials, which expectedly increase in frequency upon switch. The mean amplitude for the session, by task type, is plotted below. Ml for the entire session $=0.013$. C. Polar histogram of peak phase angles by task type for all trials across all sessions for an example rat $(n=4$ sessions). All task types indicated significant periodicity (Rayleigh test, odor only $p<1 e 118$, tone attn $p<1 e-24$, switch $p<1 e-27$, odor attn $p<1 e-31$ ), and similar distributions (Kolmogorov-Smirnov tests, all comparisons $p>0.05$ ). D. Polar histograms of correct and incorrect trials for each task type. For this example rat, incorrect trials were pooled across sessions and compared to a randomly-selected equal number of correct trials. Peak phase angle distributions were statistically 
similar between correct and incorrect trials. (Kolmogorov-Smirnov tests, all comparisons $\mathrm{p}>0.05$ ). E. Left, theta-gamma MI across rats, normalized to MI for odor only trials. One-way ANOVA, $F(2.37,9.48)=1.96, p=0.019$. Right, theta-gamma peak angle variance across rats, normalized to peak angle variance for odor only trials. One-way ANOVA, $F(2.03,8.10)=1.07, p=0.387 . n=5$ rats, $4.6+/-0.5$ sessions per rat.Error bars represent SEM.

Beta oscillations are more coherent between the mPFC and olfactory regions during an

357 intermodal attentional shift.

358 We next tested whether spectral activity between the mPFC and olfactory system might

359 become more coherent during attention. We observed enhanced coherence in the beta

360 range $(15-35 \mathrm{~Hz})$ between the mPFC and the TuS specifically during the switch blocks -

361 when the rule has been changed from tone to odor attention, but the rats have not yet

362 successfully switched their attention (Fig. 6A). For the mPFC-TuS, this elevation was

363 specific to the 1 second hold period prior to odor onset (Fig. 6B) which corresponds to

364 anticipation. Between the MPFC and the OB, we similarly observed increased coherence

365 in the beta band, but during both the hold and odor epochs (Fig. 6C-D). In contrast, no

366 changes in coherence between the OB and TuS were uncovered (data not shown).

367 Overall, these data indicate that mPFC engagement with olfactory structures is 368 upregulated during a cognitively demanding switch from auditory to olfactory selective 369 attention, suggesting a role for the mPFC in attention-dependent odor processing. 

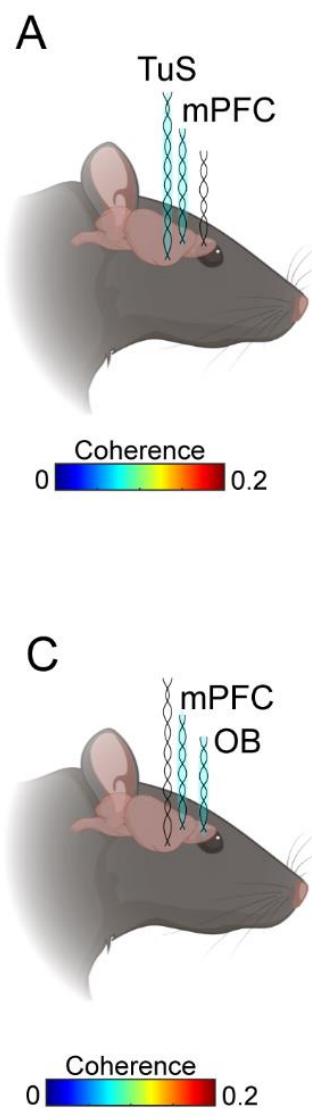

370
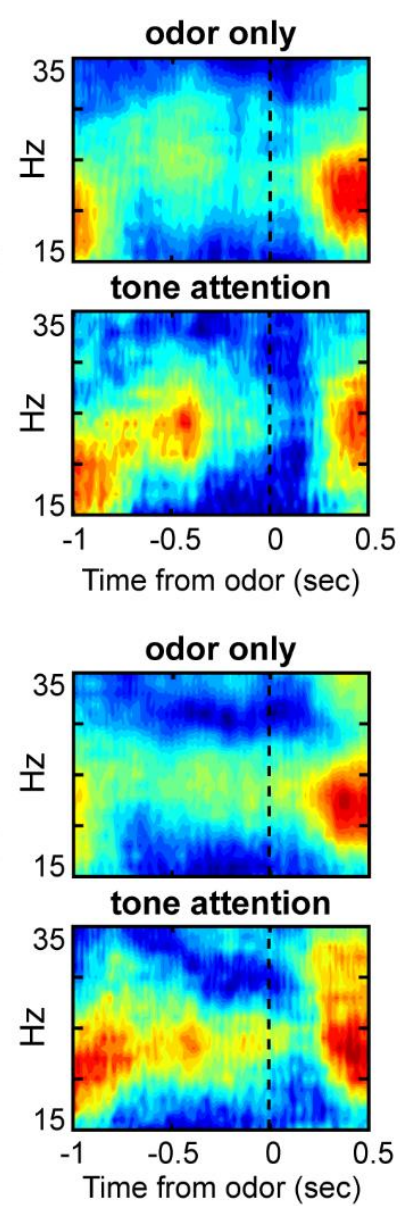
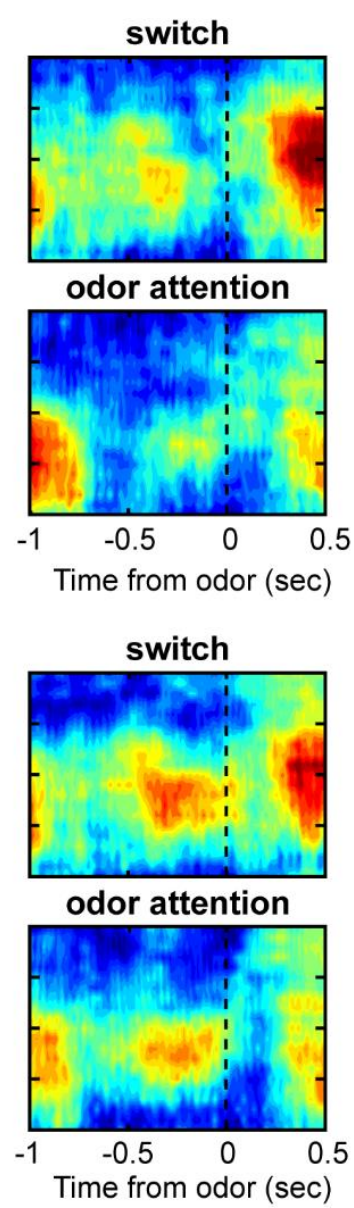

B

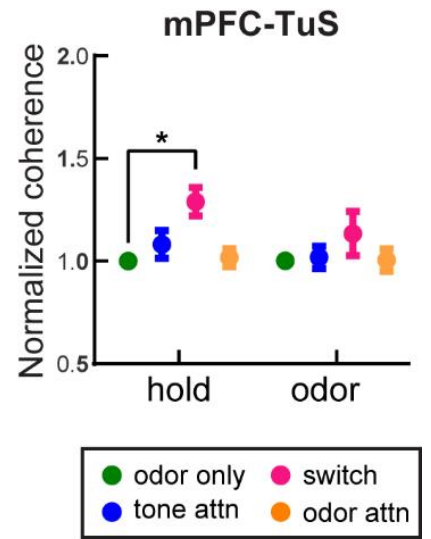

D

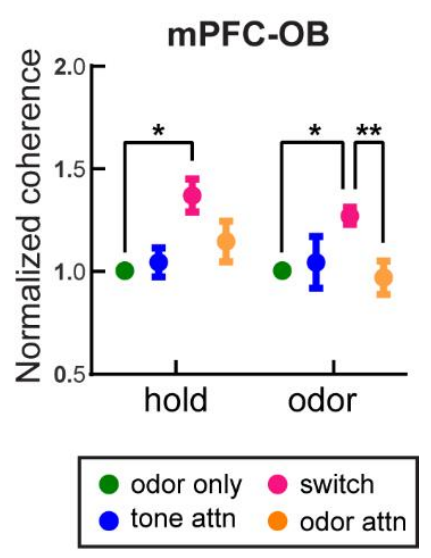

Figure 6. Beta oscillations are more coherent between the MPFC and olfactory regions during intramodal attentional shifts. A. Coherogram showing coherence between the mPFC and TuS in the beta range $(15-35 \mathrm{~Hz}$ ) for one example rat across task types ( $\mathrm{n}=3$ sessions). Nose poke begins at $-1 \mathrm{sec}$, dotted line indicates odor onset. B. Means for OT-mPFC beta coherence across all rats, normalized to odor only. $n=5$ rats, $4.6+/-0.5$ sessions per rat. 2-way ANOVA with Geisser-Greenhouse Correction, main effect of task type, $F(1.38,5.41)=6.54, p=0.041$. Error bars represent SEM. C. Coherogram showing coherence between the MPFC and the OB in the beta range $(15-35 \mathrm{~Hz})$ for one example rat across task types. Nose poke begins at $-1 \mathrm{sec}$, dotted line indicates odor onset. D. Means for OB-mPFC beta coherence across all rats, normalized to odor only. $n=5$ rats, $4.6+/-0.5$ sessions per rat. 2-way ANOVA with Geisser-Greenhouse correction, main effect of task type, $F(1.79,7.15)=13.06, p=0.005$. Error bars represent $S E M$.

$O B-m P F C$ coherence in the respiratory theta range is strongly upregulated during an intermodal attentional shift to odor attention.

Respiration, including fast investigatory sniffing, may structure theta oscillations in not only olfactory regions like the OB (e.g., (Adrian 1942; Kay and Laurent 1999; Buonviso et al. 2003)) and TuS (Carlson et al. 2014), but also the PFC (Moberly et al. 2018; Tort et 
388 al. 2018b; Biskamp et al. 2017; Bagur et al. 2021; Zhong et al. 2017). Slow wave 389 respiratory theta may serve as a carrier for synchronizing brain regions (Colgin 2013;

390 Fontanini and Bower 2006). This is particularly relevant in an odor-guided task, where 391 correct performance depends upon sampling of the odors via sniffing. We observed that 392 during the attentional switch, there was a striking increase in coherence in the theta range $393(2-12 \mathrm{~Hz})$ compared to the odor attention state (Fig. 7A-B). While a slight increase was 394 observed during the hold epoch (Fig. 7A-B), this increase was much more pronounced 395 and statistically significant during the odor sampling period (Fig. 7A-B). While our prior 396 analyses had been restricted solely to correct trials for odor only, tone attention, and odor 397 attention, we included correct and incorrect trials for all switch blocks, since this switch 398 state is defined by poor performance and behavioral flexibility, and also because this 399 allowed for the inclusion of comparable numbers of trials in the analysis (see Methods). 400 Thus, we separated trials for switch blocks only into correct and incorrect trials, discarding 401 a random selection of correct trials to match the number of incorrect trials available. This 402 revealed, counterintuitively, a greater coherence on incorrect compared to correct trials 403 (Fig 7C), suggesting that OB-mPFC theta band coherence is upregulated in contexts 404 where behavioral flexibility is required. 


$$
\text { B }
$$
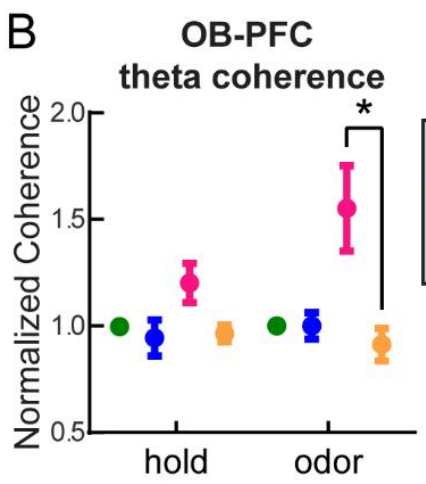

odor only
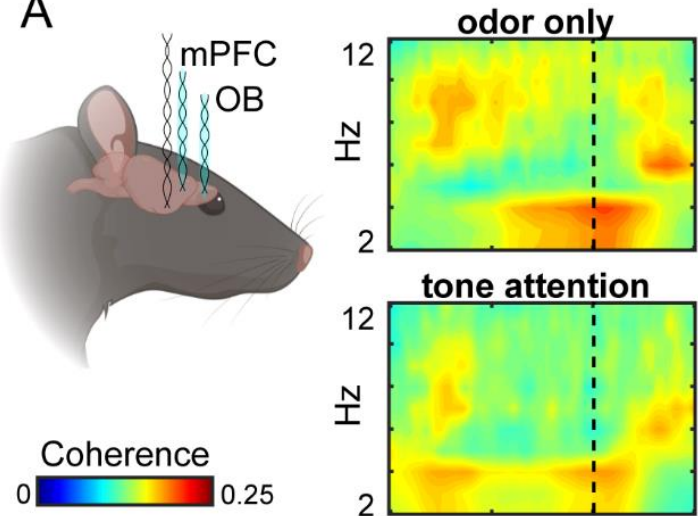

tone attention

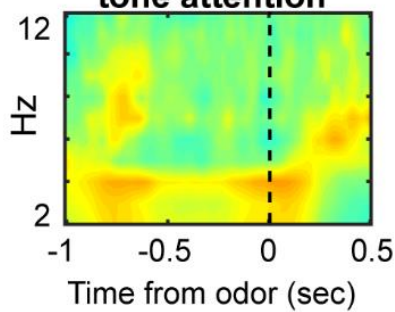

switch

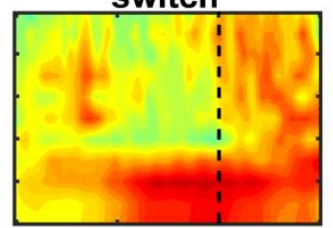

odor attention

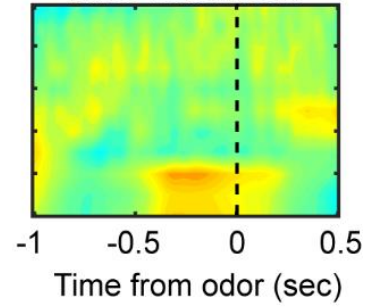

C

OB-PFC coherence

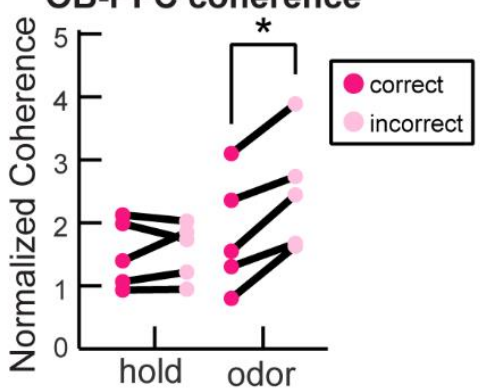

Figure 7. Olfactory bulb and medial prefrontal cortex coherence in the respiratory theta range is strongly upregulated during an intramodal attentional shift to odor attention. A. Mean coherogram across rats showing coherence in the theta range $(2-12 \mathrm{~Hz})$ across task types. B. Mean theta coherence across rats for each trial epoch and each task type. Odor only, tone attention, and odor attention trials include only correct trials from criterion performance blocks. Switch quantification includes all trials from blocks below criterion performance. 2-way ANOVA with Geisser-Greenhouse correction, main effect of task type $F(1.3,5.2)=7.07, p=0.039$. Asterisk on graph indicates results from Tukey's multiple comparison's test, * $p<0.05$. Error bars represent SEM. C. Theta coherence for correct and incorrect trials during the switch. While there were more correct than incorrect trials, randomly selected correct trials were excluded from this analysis to match the number of incorrect trials. $n=5$ rats, $4.6+/-0.5$ sessions per rat. 2-way ANOVA with Geisser-Greenhouse correction, main effect of outcome $F(1,4)=76.81, p=0.0009$. Interaction between outcome and trial epoch $F(1.56,6.24)=5.39, p=0.048$. Asterisk on graph indicates results from Sidak's multiple comparisons test, ${ }^{*} \mathrm{p}<0.05$.

424 Sniffing behavior in rodents is influenced by many factors including wakefulness, the

425 stimulus being sampled, and motivational state (Clarke and Trowill 1971; Ikemoto and 
426 Panksepp 1994; Wesson et al. 2008; Kepecs et al. 2007; Rojas-Líbano and Kay 2012;

427 Lefèvre et al. 2016). As discussed above, whether passive (respiration) or active

428 (sniffing), this behavior subsequently shapes neural activity throughout the brain (Adrian

429 1942; Macrides et al. 1982; Vanderwolf 1992; Colgin 2013; Fontanini and Bower 2006;

430 Verhagen et al. 2007; Buonviso et al. 2003; Carey et al. 2009; Jordan et al. 2018;

431 Shusterman et al. 2011; Sobel and Tank 1993; Spors et al. 2006). We reasoned that if

432 rats adjusted their sniffing when faced with the demand to selectively attend to odors, this

433 could potentially account for the changes in OB-mPFC theta coherence. No prior work

434 has assessed sniffing strategies of rodents during olfactory selective attention. To test

435 this, we trained a separate cohort of rats to perform the CAT before implanting

436 thermocouples in their nasal cavities, allowing us to monitor sniffing behavior by

437 measuring temperature changes (airflow) within the nasal cavity (Fig. 8A-B). Unlike

438 humans, who respond to changing attentional demands by modifying both the depth and

439 timing of respiration (Plailly et al. 2008; Arabkheradmand et al. 2020), rodents most

440 dramatically employ changes in sniffing frequency during odor active sampling (Cenier et

441 al. 2013; Wesson et al. 2009; Kepecs et al. 2007). Therefore, we quantified sniffing

442 frequency specifically during the hold and odor periods. As illustrated by the example

443 session from one rat in Fig. 8C, we observed no clear changes in sniffing behavior across

444 task types (Fig. 8C-D). Instead, the rats displayed a highly stereotyped pattern of sniffing

445 behavior, suggesting that reaching high proficiency on the CAT results in their

446 development of a sensorimotor program that is implemented on each trial, regardless of

447 current attentional demand (Fig. 8C, bottom). This was the case across all rats. Although

448 we observed a slight decrease in sniffing frequency specifically during the hold period 
449 throughout a session on average (Fig. 8D, F), this was confined to the hold period as the

450 rats anticipated odor arrival and sniffing frequency during the odor sampling period

451 remained remarkably constant throughout the sessions for 2/3 rats (Fig. 8D, F). Given

452 the lack of changes in sniffing frequency by task type, we investigated whether the timing

453 of sniffs during the odor period were more intentional in relation to odor onset when

454 animals were faced with attending to odor. We examined the time to the first sniff across

455 task types and found no difference, suggesting that sniff timing relative to odor onset is

456 independent of attentional demands (Fig. 8E). We also examined sniffing frequency on

457 correct versus incorrect trials during the switch blocks (Fig. 8G) yet did not identify

458 differences in sniffing frequency, providing further evidence that changes in sniffing

459 behavior do not likely account for modulations in OB-mPFC coherence that we

460 uncovered, which were correlated with trial outcome. Together, these data indicate that

461 sniffing strategies in rats are resilient to enhanced attentional demand, providing evidence

462 for covert (rather than overt) olfactory attention in rodents. 

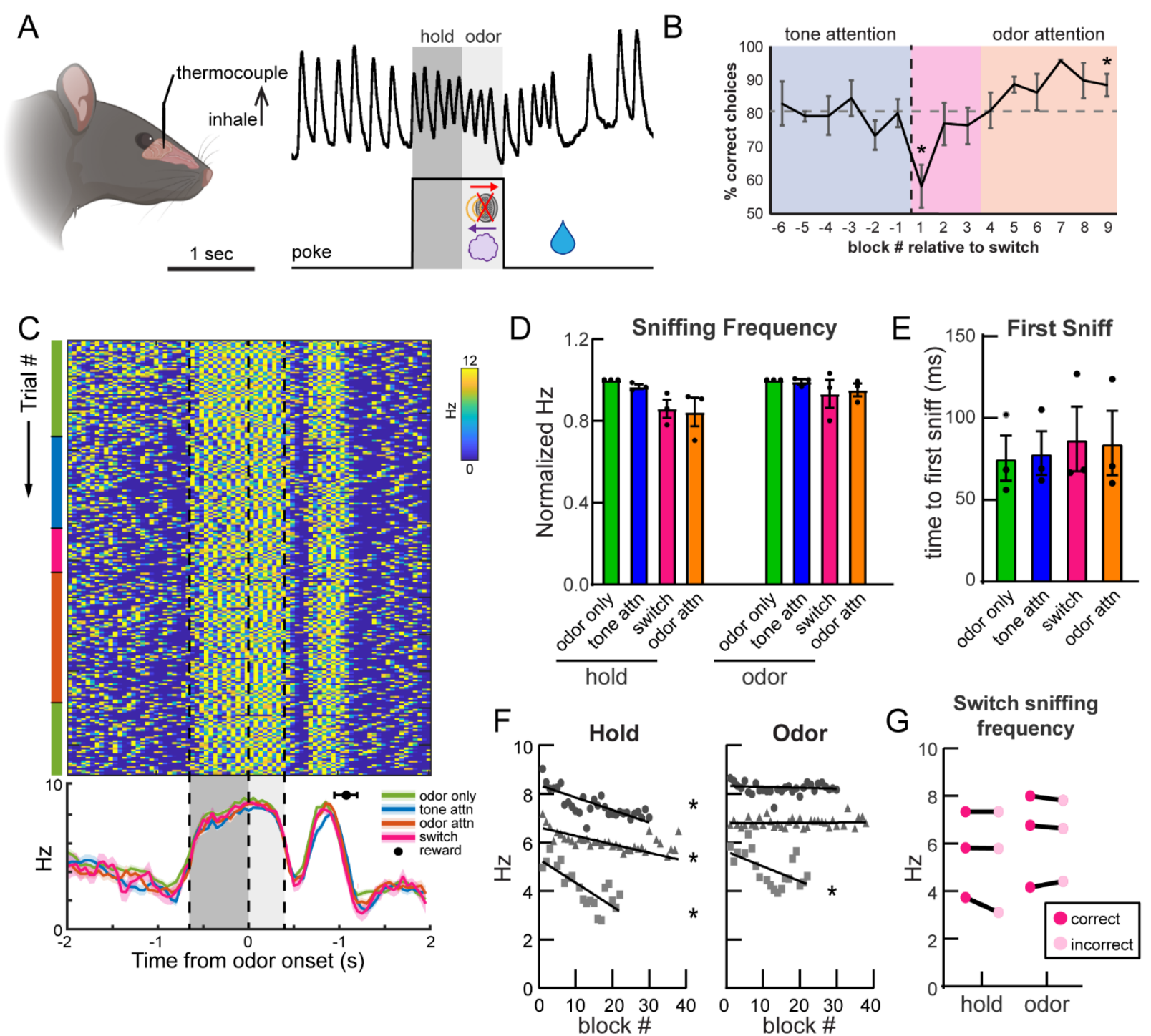

Figure 8. Rats maintain highly stereotyped sniffing strategies despite increased attentional demands. A. Sample trace of thermocouple signal from rat nasal cavity on a single trial. $600 \mathrm{~ms}$ hold and $400 \mathrm{~ms}$ odor epochs are indicated by dark and light gray shading, respectively. B. Behavioral performance. Block -1 vs. 1 paired, two-tailed t-test, $p=0.049$. Block 1 vs. 9 paired, two-tailed t-test, $p=0.026$. C. Instantaneous sniff frequency for one session, from one example rat (rat 137). Colored bars on the left-hand side indicate the current task type. Dotted lines and light and dark gray shading represent hold and odor epoch respectively. Mean sniffing frequency \pm SEM for each task type is plotted below, with the black circle indicating the mean time of reward acquisition $( \pm S E M)$. D. Sniffing frequency means within each trial epoch. 2-way ANOVA with Geisser-Greenhouse correction, main effect of trial epoch $F(1,2)=29.47, p=0.032$. No main effect of task type, $F(1.04,2.09)=3.2, p=0.21$. Error bars represent SEM. E. Time to the first sniff following odor onset. One-way ANOVA with Geisser-Greenhouse correction, $F(1.19,2.38)=1.91$, $p=0.29$. Error bars represent SEM. Similar results were seen when calculating time to second and third sniffs, as well as intervals between them (data not shown). F. Correlations between block \# of the session and sniffing frequency. Rat 137 (circles) hold: $R^{2}=0.33, F(1,111)=54.46, p<0.0001$, odor: $R^{2}=0.009, F(1,111)=1.03, p=0.31$. Rat 138 (squares) hold: $R^{2}=0.61, F(1,18)=28.61$, $p<0.0001$, odor: $R^{2}=0.32, F(1,18)=8.406, p=0.009$. Rat 139 (triangles) hold: $R^{2}=0.3$, $F(1,168)=73.14, p<0.0001$, odor: $R^{2}=0.0004, F(1,168)=0.07, p=0.79$. G. Sniffing frequency for 
482

483

484

485

486

487

488

489

490

491

492

493

494

495

496

497

498

499

500

501

502

503

504

505

506

507

correct and incorrect trials in switch blocks only. 2-way ANOVA with Geisser-Greenhouse correction, main effect of trial epoch, $F(1,2)=57.65, p=0.017$. No main effect of trial outcome. $n=3$ rats, $3.5 \pm 2.5$ sessions per rat.

\section{Discussion}

Here we used anatomical, behavioral, and physiological approaches to demonstrate integration of the mPFC with the olfactory system in the context of selective attention to odors. We show that mPFC neurons in the PrL and IL subregions directly and preferentially target the mTuS compared to other olfactory regions, suggesting that they are well-positioned to exert influence on olfactory processing via the mTuS. We then used a physiological and behavioral approach to demonstrate local and interregional effects of attention on network activity within and between the mPFC and olfactory regions, including the $\mathrm{OB}$ and TuS. Finally, we found that olfactory sampling behavior is resilient to attentional demand, indicating that olfactory attention may be an "covert" rather than "overt" process. Together, this work adds to a growing body of literature on the possible mechanisms underlying cognitive modulation of olfactory processing and thus perception.

\section{Insights into MPFC connectivity with the olfactory system.}

Our tracing experiments uncovered previously unappreciated aspects of mPFC connectivity with the olfactory system. We found that the PrL and IL most densely innervate the mTuS compared with other olfactory regions. By using a combinatorial AAV approach, where Cre expression driven by the CaMKII promotor permits expression of synaptophysin-eGFP/-mRuby, we were able to identify this pathway as excitatory while confidently attributing fluorescence in the TuS (and PCX) to synaptic terminals (primarily in layers 2 and 3) rather than fibers of passage (Fig. 1). We further demonstrated that 
508 among PFC subregions, the PrL and IL provide the most projections to the TuS, with the

$509 \mathrm{MO}$ coming in third, and these projection neurons mostly reside in layer 5 (Fig. 2). Our

510 data are in agreement with earlier tracing work which established that rat mPFC neurons

511 project throughout the brain, including in the TuS (Vertes 2004), and a recent review

512 proposing that the PrL, IL and MO be grouped together as the ventromedial PFC, based

513 upon their connectivity (Le Merre et al. 2021). Our results expand upon previous literature,

514 which used anterograde phaseolus vulgaris-leucoagglutinin tracing (Vertes 2004), by

515 contributing (1) specificity and certainty regarding the specific layers of mPFC $\rightarrow$ TuS

516 synapses and (2) clarity about at least one mPFC cell type. mPFC glutamatergic neurons

517 modulate their firing during sustained attention (Kim et al. 2016) and encode task rules

518 during an intermodal attention task (Rikhye et al. 2018), suggesting that TuS-projecting

519 glutamatergic mPFC neurons are positioned to influence olfactory processing during

520 attentionally-relevant behavioral events. Future work illuminating the more specific

521 identities of these TuS projecting mPFC neurons (e.g., via transcriptomics) will be

522 important for disambiguating their specific circuitry and possible contribution to olfactory

523 attention.

524

525 Prefrontal-olfactory network engagement during selective attention.

526 Our multisite LFP recordings during attentional performance uncovered many changes in

527 network activity which expand our appreciation for how the olfactory system is shaped by

528 cognitive state. There are several especially notable outcomes we discuss here.

529 First, while we know that the mPFC is crucial for attention, no studies have 530 monitored mPFC network activity during olfactory attention, leaving a major void in our 
531 understanding of how the mPFC engages with the olfactory system. Because the mPFC

532 is integral for some forms of attention, we predicted it may be recruited during olfactory

533 attention. In support of this, we observed elevated gamma power in the mPFC during

534 odor-directed attention relative to tone attention (Fig. 4). The mPFC is certainly not an

535 olfaction-specific structure, though it is engaged by odor-guided tasks requiring learning

536 (Wang et al. 2020) and high working memory capacity (De Falco et al. 2019). This

537 elevation in gamma power does not likely reflect increased reward confidence, as

538 behavioral performance was comparable across task types (Fig. 3C). It is interesting to

539 consider whether the mPFC of rodents is predisposed to favor and prioritize olfactory

540 information more so than other sensory stimuli. Nevertheless, these findings exhibit

541 engagement of the mPFC during odor-directed attention, providing support for its

542 inclusion in an olfactory attention network.

544 Olfactory attention enhances power of OB gamma oscillations.

545 Our work is the first to monitor OB activity during selective attention. We found that

546 attention powerfully shapes OB activity, which implies that odor information received by

547 structures downstream from the OB, including the TuS, is subject to attention-dependent

548 modulation. Specifically, we observed increased power of low gamma oscillations (40-60

$549 \mathrm{~Hz}$ ) in the OB during odor-directed attention as compared to odor only discriminations

550 (Fig. 4). Additionally, we observed elevated power of high gamma oscillations $(60-80 \mathrm{~Hz})$

551 while rats attempted to switch their attention from tones to odors (Fig. 4). While increased

552 gamma power in the $\mathrm{OB}$ has been associated with successful discrimination of

553 perceptually similar vs. dissimilar odors (Beshel et al. 2007), our findings indicate that 
554 similar effects can be observed when the odor discrimination is simple/coarse, but the

555 attentional demand is high. Interestingly, elevated low gamma power during odor

556 attention was evident during both the hold and odor epochs, while elevated high gamma

557 power during switch blocks was isolated to the odor sampling period (Fig. 4B). High and

558 low gamma oscillations are considered distinct phenomena in the $\mathrm{OB}$, and are believed

559 to have mechanistically unique origins (Kay 2003), so it is perhaps not surprising to

560 observe modulation of these frequency bands during different attentional demands. Low

561 gamma oscillations are believed to arise from inhibition between local interneurons, are

562 unstructured relative to the sniff cycle, and are functionally mysterious, though they have

563 been observed in states of engaged quiescence (Kay 2003). Our data thus support a

564 potential role for low gamma oscillations in attentionally demanding odor discriminations,

565 though future work is needed to fully appreciate the mechanisms of this.

566 In contrast, high gamma is structured to the sniff cycle, and is generated by local

567 excitatory-inhibitory interactions (Schoppa 2006; Neville and Haberly 2003; Halabisky

568 and Strowbridge 2003; Lepousez and Lledo 2013). Disruption of high gamma oscillations

569 in the OB impairs odor discrimination, suggesting their importance for basic aspects of

570 odor perception (Lepousez and Lledo 2013). While the mechanisms by which they may

571 be modulated are unclear, one compelling proposition is that neuromodulators, including

572 acetylcholine and noradrenaline, influence excitatory-inhibitory interactions in the OB

573 (Kay et al. 2009). This is of particular interest given the role of these neuromodulators in

574 states of attention and arousal (Sara 2009; Yu and Dayan 2005). Our observation that

575 high gamma power elevations during the switch are confined to the odor sampling period

576 is consistent with these mechanistic underpinnings and suggests specific changes in the 
577 nature of odor processing as one undergoes a cognitively demanding switch to odor

578 attention.

579 As mentioned, high frequency gamma in the $\mathrm{OB}$ is consistently aligned with the 580 respiratory cycle, which was evident in our PAC analysis (Fig. 5). Recent work 581 demonstrated that $\mathrm{OB}$ theta-high gamma PAC is strengthened as mice learn to 582 discriminate odors in a go-no go task, specifically for the go stimulus, suggesting that 583 PAC may support olfactory behavior (Losacco et al. 2020a) and leading us to test whether 584 attention employs (or perhaps just simply influences) OB PAC. Our results uncovered 585 highly consistent theta-gamma PAC in the OB across attentional demands, and much 586 weaker coupling between theta and beta oscillations, leading us to focus on theta-gamma 587 PAC. However, we did not observe a decrease in PAC when expert rats completed trials 588 incorrectly (Fig. 5D), suggesting that perhaps PAC is not necessary to successfully 589 discriminate coarse odor pairs, like the ones we used herein. One possible explanation 590 for this difference is that in 2-alternative choice tasks, like the CAT, both stimuli are 591 assigned positive valence, while in go no-go tasks like that used by (Losacco et al. 592 2020a), one stimulus loses positive valence upon learning. Throughout a single session 593 of the CAT, odors temporarily lose their reward-predictive value during tone attention, but 594 it is quickly regained (e.g., Fig. 3C). Our data indicate that OB PAC, in rats who have 595 been shaped to expert level on the same odor discrimination over many weeks, is resilient 596 to a temporary lapse in positive odor valence, and perhaps supports flexible behavior 597 supporting attentional switches. 
600 Beta synchrony integrates MPFC activity within the olfactory network.

601 Our data are the first to show functional coupling between the mPFC and olfactory regions

602 during attentional demands - specifically during an intermodal attentional shift to odors

603 (Fig. 6). Beta oscillations are considered an important mechanism by which long-range

604 communication can occur between brain regions (Spitzer and Haegens 2017; Kopell et

605 al. 2000), and further, are implicated in top-down control of attention (Richter et al. 2017;

606 Sacchet et al. 2015). We observed elevated beta coherence between the mPFC and the

607 TuS as rats attempted to switch their attention from tones to odors (Fig. 6A-B). In the 608 context of our finding that the mPFC and the TuS are connected via a unidirectional

609 monosynaptic pathway (Figs. 1-2), these data suggest that communication between the 610 mPFC and TuS is strengthened during attentional shifts. Indeed, ventral striatum-

611 projecting mPFC neurons are implicated in cognitive flexibility by integrating feedback

612 from trial outcomes (Spellman et al. 2021), suggesting that the mPFC $\rightarrow$ TuS pathway 613 could engage in the same processes. Our findings provide further support for the 614 hypothesis that interareal beta oscillations may be a mechanism by which information 615 about behavioral context is conveyed from higher-level cortex to lower-level sensory 616 areas (Bressler and Richter 2015; Wang 2010; Kay and Freeman 1998), and are the first

617 to demonstrate that this concept is applicable to the olfactory system, which possesses 618 unique anatomical organization.

619 In addition to enhanced mPFC-TuS coherence, we also observed enhanced beta 620 band coherence between the mPFC and the OB (Fig. 6C-D), raising the intriguing 621 possibility that prefrontal influence on olfactory processing could begin as early as the 622 OB. While the OB and mPFC are not connected monosynaptically (Fig 1), they are 
623 intermediately connected via bidirectional connectivity with the AON, a pathway known to

624 drive coherence between these structures (Moberly et al. 2018). Additionally, these two

625 regions both receive inputs from key neuromodulatory nuclei (Santana and Artigas 2017;

626 Devore and Linster 2012; McLean et al. 1989; Rothermel et al. 2014; Passetti et al. 2000;

627 Devoto et al. 2005; Zaborszky et al. 1986), which may influence the power of beta

628 oscillations in the OB, perhaps by modifying granule cell excitability (Osinski et al. 2018).

629 This raises the possibility that neuromodulators may enable mPFC-OB coherence via

630 simultaneous phasic input to both the mPFC and OB. Cholinergic input increases

631 (Passetti et al. 2000; Himmelheber et al. 2000) and modulates mPFC firing in the context

632 of attention (Gill et al. 2000), and powerfully alters the encoding of odors in the OB

633 (Chaudhury et al. 2009; Devore and Linster 2012; Ogg et al. 2018; D'Souza and

634 Vijayaraghavan 2014). Indeed, lesioning of cholinergic nuclei results in reduced beta

635 synchrony and increased attentional errors in rats (Ljubojevic et al. 2018), indicating at

636 role for cholinergic modulation in attention-related interregional synchrony. Whether

637 cholinergic mechanisms contribute to attention-driven beta synchrony between the mPFC

638 and $\mathrm{OB}$ as we observed is an important future question.

640 Olfactory sampling is resilient to attentional demand.

641 Odor perception requires the inhalation of an odor, and in rodents this occurs by

642 means of rhythmic inhalation and exhalation of air through the nose in the theta rhythm

643 (Welker 1964; Youngentob et al. 1987; Wesson et al. 2008; Kepecs et al. 2007). The work

644 herein is the first to investigate the influence of olfactory selective attention on sniffing

645 behavior in a rodent. This question is of great interest and importance, since theta 
646 oscillations in both olfactory regions and beyond are profoundly shaped by respiration

647 (Adrian 1942; Macrides 1975; Vanderwolf 1992; Tort et al. 2018a; Colgin 2013; Zhang et

648 al. 2021; Fontanini and Bower 2006; Kay and Laurent 1999; Buonviso et al. 2003; Miura

649 et al. 2012). Especially relevant to our work, low frequency respiration during freezing

650 behavior can drive strong coherence between OB and mPFC activity, further supporting

651 functional connectivity between these networks (Moberly et al. 2018; Bagur et al. 2021).

652 Rodents structure their sniffing in manners influenced by motivation, behavioral

653 task structure, and the sensory stimulus itself (Wesson et al. 2008; Kepecs et al. 2007;

654 Clarke and Trowill 1971; Ikemoto and Panksepp 1994; Rojas-Líbano and Kay 2012;

655 Lefèvre et al. 2016). While some findings suggest that sniffing strategies do not change

656 in the face of increased perceptual difficulty (Wesson et al. 2009; Uchida and Mainen

657 2003), we hypothesized, based on our finding of increased theta synchrony between the

658 OB and mPFC (Fig. 7), that enhanced attentional demand may influence sampling

659 strategy. For instance, a rat might increase sniffing frequency during odor sampling when

660 attention to odors versus attending to tones. We found that rats' sniffing strategies were

661 remarkably resilient to shifting attentional demands, remaining stereotyped as rats flexibly

662 switched their attention from the auditory to olfactory modality (Fig. 8). This is in contrast

663 to some findings in humans, indicating that humans alter the timing and depth of their

664 inhalations during odor anticipation and/or attention (Arabkheradmand et al. 2020; Plailly

665 et al. 2006). Interestingly, humans also structure their inhalations relative to task structure

666 even when the task is not olfactory in nature, pointing to a role for respiration in structuring

667 and supporting behavioral performance overall (Perl et al. 2019). This idea, along with

668 our findings, together raise the intriguing possibility that rhythmic sniffing may even 
669 enhance perception of other stimulus modalities (e.g., auditory), perhaps via cross-modal

670 entrainment (Bauer et al. 2021; Lakatos et al. 2019).

671 It is interesting to consider sensory sampling via sniffing as analogous to saccadic

672 eye movements (Uchida et al. 2006), which contribute to rhythmic attentional sampling in

673 the visual system (Fiebelkorn and Kastner 2019; VanRullen 2016). In the visual system,

674 attention is regarded as overt when it is accompanied by saccadic eye movements to a

675 target and covert when the eyes remain fixated on a central point (Posner et al. 1980).

676 The investigation of these different modes of attention and their underlying networks has

677 spanned decades (Posner 2016). While these two processes engage similar brain

678 networks (Rizzolatti et al. 1987; Corbetta 1998), suggesting that they may not actually be

679 separate, other work suggests different populations of neurons within these networks may

680 support each type of attention (Thompson et al. 2005). Analogously, our observation of

681 covert olfactory attention (i.e. olfactory attention that occurs in the absence of attention-

682 specific changes in sniffing behavior; Fig. 8) does not necessitate that olfactory attention

683 is always covert (i.e. sniffing is unaffected), and perhaps different behavioral contexts

684 might engage different olfactory attentional frameworks.

685

686 Conclusion.

687 Taken together, our data support a model of olfactory attention in which the mPFC

688 integrates with olfactory regions at early (OB) and later (TuS) stages of odor processing

689 to form an olfactory attention network. This network encompasses local attention-

690 dependent changes in activity within the OB and mPFC, as well as strengthening of

691 interregional coupling between the mPFC-OB and mPFC-TuS. Our data suggest that 
692 changes in sniffing do not drive these effects, highlighting that odor-directed attention, at

693 least in this context, is orchestrated by top-down mechanisms, as opposed to 'bottom-up'

694 influences (from odor sampling). Overall, these findings begin to reveal an olfactory

695 attention network and bring us closer to understanding how the brain affords the ability to

696 selectively attend to odors. 


\section{Materials and Methods}

698 Animals

699 Adult, male Long-Evans rats were obtained from Charles River (Wilmington, MA) and

700 Envigo (Indianapolis, IN) and maintained in the University of Florida vivarium on a 12:12

701 light:dark cycle, with food and water provided ad libitum until water restriction for

702 behavioral shaping began. All experiments were conducted in accordance with NIH

703 guidelines and were approved by the University of Florida Institutional Animal Care and

704 Use Committee.

705

Surgical procedures

707 For all surgical procedures, rats were maintained on $4-1 \%$ isofluorane in $1.5 \mathrm{~L} / \mathrm{min}_{2}$ and

708 placed in a stereotaxic frame. The scalp was shaved and cleaned with betadine and $70 \%$

709 ethanol. Analgesia in the form of meloxicam was administered ( $5 \mathrm{mg} / \mathrm{kg} \mathrm{s.c.)}$ and the local

710 anesthetic marcaine (5 mg/kg s.c.) was given prior to the cranial incision. A cranial incision

711 was made and the skin was retracted using hemostats.

712 For viral injections, a craniotomy was then drilled over the region of interest, and a

713 glass micropipette containing AAV was slowly lowered into region of interest. For

714 anterograde mPFC injections (Fig. 1), $100 \mathrm{~nL}$ of a 50/50 mixture of Cre-dependent

715 synaptophysin virus (Ef1a-DIO-Synaptophysin-mRuby in IL; Ef1a-FLEX_Synaptophysin-

716 GFP in PrL; both generous gifts from Dr. Marc Fuccillo, Univ of Pennsylvania) (Herman

717 et al. 2016) and CaMKII-Cre virus (pENN-AAV9-CaMKII-Cre-SV40; Addgene,

718 Watertown, MA; 105558-AAV9, titer $1 \times 10^{\wedge} 13 \mathrm{vg} / \mathrm{mL}$ ) was injected into the $\mathrm{IL}$, then the

719 PrL, at a rate of $2 \mathrm{~nL} / \mathrm{sec}$. For retrograde mPFC injections (Fig. S1), $200 \mathrm{~nL}$ total of AAVrg- 
720 hSyn-GFP (Addgene 50465-AAVrg; titer 7x10^12 vg/mL) was unilaterally injected at a

721 rate or $2 \mathrm{~nL} / \mathrm{sec}$ into the $\mathrm{mPFC}(100 \mathrm{~nL}$ in $\mathrm{IL}$, followed by $100 \mathrm{~nL}$ in PrL). For TuS

722 injections, $200 \mathrm{~nL}$ of AAVrg-hSyn-GFP (Addgene 50465-AAVrg; titer 7x10^12 vg/mL) was

723 injected unilaterally at a rate of $2 \mathrm{~nL} / \mathrm{sec}$. In all cases, after waiting 5 minutes, the pipette

724 was slowly withdrawn from the brain, the craniotomy was sealed with dental wax, and the

725 incision was sutured.

726 For electrode implants, the skull was scrubbed with $3 \% \mathrm{H}_{2} \mathrm{O}_{2}$ and covered with a

727 thin layer of cyanoacrylate (Vetbond, 3M). Craniotomies were drilled over each brain area

728 of interest, plus 3 craniotomies for $0-80$ stainless-steel screws to aid in anchoring the

729 dental cement. After drilling craniotomies over each brain area of interest, the bipolar

730 stainless-steel electrodes (0.005-in outer diameter, Teflon coated to 0.007-in outer

731 diameter) were lowered into the brain and secured with a small amount of dental cement

732 before moving on to the next electrode. Once all wires were placed and secured, an

733 electrical interface board (EIB) (Open Ephys, Cambridge, MA) fitted with a 32 channel

734 connector (Omnetics, Minneapolis, MN) was lowered over the skull, and the electrode

735 wires were secured to the desired channels using gold pins. After the stainless-steel

736 ground wire was secured to a skull screw with conductive silver paint, the whole assembly

737 was secured with dental cement.

738 For thermocouple implants (Wesson 2013; Uchida and Mainen 2003), following

739 skull preparation as above, a craniotomy was made in the nasal bone $(0.9 \mathrm{~mm}$ lateral

740 from midline) and a thermocouple wire was lowered $3 \mathrm{~mm}$ into the nasal cavity and

741 secured with dental cement. Then, as for the electrode implants, an EIB with a 32-channel

742 Omnetics connector was lowered over the skull, the thermocouple leads secured with 
743 gold pins, and a ground wire secured to a skull screw before the whole assembly was

744 secured with dental cement.

745 Following surgery, rats were returned to their home cages to recover on a heating

746 blanket. The rats received post-operative analgesia for at least 3 days mixed with a

747 palatable gel (5 mg/kg meloxicam in Medigel, ClearH2O, Westbrook, ME). Electrode

748 implanted rats were implanted prior to the beginning of behavioral shaping. Thermocouple

749 implanted rats were shaped prior to surgery and were allowed full water access for at

750 least 24 hours prior to surgery. All rats were allowed to recover for at least 5 days before

751 beginning or restarting water restriction.

752

753 Perfusion and histology

754 For anterograde mPFC viral injections (Fig. 1), rats were perfused 2-4 weeks following

755 injection. For retrograde mPFC (Fig. S1), and TuS viral injections (Fig. 2), rats were

756 perfused 2 weeks following injection. All rats were overdosed with Fatal-Plus and

757 perfused with cold $0.9 \% \mathrm{NaCl}$ followed by cold $4 \%$ formalin. Brains were dissected and

758 stored in $10 \%$ formalin in $30 \%$ sucrose prior to sectioning. Alternate 40 um sections were

759 collected with a sliding microtome and stored in Tris-buffered saline with $0.03 \%$ sodium

760 azide. For electrode implanted rats, sections were mounted on gelatin subbed slides and

761 stained with $0.1 \%$ cresyl violet to confirm electrode locations.

762

763

Image acquisition and quantification

764 Brain areas of interest were identified using the rat brain atlas (Paxinos and Watson

765 1997). Images were acquired with a Nikon Eclipse Ti2e fluorescent microscope at 20x 
magnification using a Nikon 16 MP DS-Qi2 monochrome CMOS camera. For all tracing experiments, successful targeting of the desired subregion was confirmed, and injections with spillover into surrounding regions were excluded. For anterograde mPFC injections

(Fig. 1), if one of the two injections was on target, we analyzed only that region and disregarded the other. Overall, we analyzed 8 rats with on target $\operatorname{PrL}$ injections and 5 rats

771 with on target IL injections, with 3 rats having both $\operatorname{PrL}$ and IL quantified. From these rats, 772 images for quantification were acquired as follows: for the TuS, 11 images per rat, evenly

773 spanning $2.7 \mathrm{~mm}$ anterior $-0.8 \mathrm{~mm}$ posterior Bregma. For the PCX, 17 images per rat 774 were quantified, evenly spanning $3.7 \mathrm{~mm}$ anterior $-4.8 \mathrm{~mm}$ posterior Bregma. For the 775 AON, 6 images per rat were quantified, evenly spanning 5.7mm-2.7mm anterior Bregma.

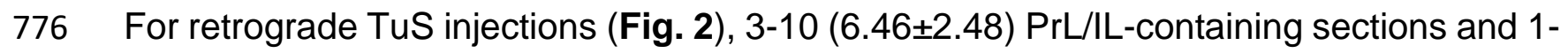

$7776(3.9 \pm 1.46)$ OFC-containing sections were imaged ( $n=6$ rats).

After acquiring images, ROls were drawn around each area of interest and

779 fluorescent puncta or cell bodies were detected using semi-automated counting 780 algorithms created within NIS elements software (Nikon) based on their fluorescence 781 intensity and size. Cell or puncta counts were then normalized to the ROI area for

782 comparison across regions. For layer-specific quantification (Fig. 2E), custom MATLAB

783 code was used to determine the layer in which each counted cell resided. The medial and

784 lateral TuS were defined as the medial and lateral third of the TuS, to ensure clear

785 separation between the regions. In puncta quantification, we initially differentiated 786 between the anterior and posterior PCX, which was divided based on the presence or 787 absence respectively, of the lateral olfactory tract. Because we observed no differences 788 in puncta between the anterior and posterior PCX for PrL (paired, two-tailed t-test, $\mathrm{p}=0.32$ ) 
789 or IL (paired, two-tailed t-test, $\mathrm{p}=0.12$ ), we combined them for the data and analyses

790 shown in Fig. 1.

791

792 Olfactory and auditory stimuli

793 The odors used for all experiments were isopentyl acetate and limonene(-), obtained at

794 their highest available purity (Sigma, St. Louis, MO), and diluted in mineral oil (Sigma) to

$795 \quad 0.5$ Torr so that they possessed equal vapor pressures. Odors were delivered through

796 independent lines via an air-dilution olfactometer at 2 L/min via a custom 3-D printed

797 nose-poke port. The auditory stimulus was a $2.5 \mathrm{kHz}$ tone $(\sim 70 \mathrm{~dB})$ generated with a piezo

798 speaker (RadioShack, Boston, MA).

799

800

Carlson Attention Task

801 Rats were water restricted to no less than $15 \%$ of their initial body weight and were 802 shaped on the Carlson Attention Task (CAT) as described in detail previously (Carlson et

803 al. 2018). Briefly, rats were first shaped on single-modality 2-alternative choice (2-AC) 804 tasks in blocks of 20 trials, starting with tone-on/tone-off 2-AC, then odor A/odor B, before 805 learning the multi-modal attention task. In the final task, rats initiated a trial by nose poking 806 in a center port. They were required to hold for 1 second (for LFP rats) or $600 \mathrm{~ms}$ (for 807 sniffing rats) before stimulus delivery, and were then required to remain for at least 400 808 ms for stimulus delivery (the prolonged hold period for the rats contributing LFP data was 809 implemented to provide a sufficient window for subsequent analyses). After leaving the 810 center port, the rats had 4 seconds to make a choice at either the left or right port. Correct 811 choices were rewarded with $15 \mathrm{uL}$ of $2 \mathrm{mM}$ saccharin in water, and incorrect choices were 
812 unrewarded. If no choices were made in the 4 second window, the trial was recorded as

813 an omission. After the 4 second window, an additional $1 \mathrm{sec}$ inter-trial interval (ITI) was

814 implemented which was reset by a nose poke during that second. Thus, across all trials

815 the rats were out of the center port for one full second prior to their trial-initiating poke.

816 Sessions began with 3 blocks of odor only, in which there were no competing tone cues.

817 After completing 3 blocks at $\geq 80 \%$ correct, the rats began receiving simultaneous

818 olfactory and auditory cues, and were required to complete 6 blocks of tone attention

819 (attending tones, and ignoring odors) at $\geq 80 \%$ correct. After this, an uncued rule change

820 occurred, requiring the rats to now attend odors, and ignore tones. After 6 blocks at $\geq 80 \%$

821 correct on odor attention, the rats completed 3 more blocks of odor only at the end of the

822 session. Odor only blocks were included at the beginning and end of the session to

823 neutralize any potential effects of motivation. For all task types, trial combinations were

824 pseudorandomly presented, such that equal numbers of each trial type were given in each

825 block of 20 trials.

826 For LFP recordings, this shaping process occurred over the course of 33-46 (40.8

$827 \pm 2.3)$ sessions, resulting in expert rats who had switched their attention from tones to

828 odors 8-11 (9.8 \pm 0.6$)$ times prior to the final recorded sessions included in our data

829 analysis. Each rat contributed 3-6 (4.6 \pm 0.5$)$ sessions of expert performance to the

830 analysis. For sniffing recordings, shaping occurred over 63-72 (67.3 \pm 2.4$)$ sessions, rats

831 switched their attention 11-17 (13.6 \pm 1.8) times before recorded sessions, and

832 contributed 1-6 $(3.5 \pm 2.5)$ sessions of expert performance to the analysis. Shaping with

833 the rats used for sniffing took more sessions for them to reach expert performance since 
834 they were delayed in learning/performance due to surgical implantation of thermocouples

835 mid task acquisition.

837 Data acquisition

838 LFPs from all electrodes were digitized using an RHD 2132 headstage (Intan

839 Technologies, Los Angeles, CA), amplified using a PZ5 amplifier (Tucker-Davis

840 Technologies, Alachua, FL), and acquired at $3 \mathrm{kHz}$ using OpenEx and an RZ2 BioAmp

841 processor (Tucker-Davis Technologies). Tethering of the rats to the PZ5 occurred via a

842 flexible ultralight tether with a commutator in-line to allow free movement. Entrances to

843 the left, right, and center ports were detected by infrared beam breaks and acquired at

$844380 \mathrm{~Hz}$. Behavioral and stimulus delivery events were simultaneously recorded in

845 OpenEx using the RZ2 BioAmp processor, allowing for synchrony between the behavioral

846 and neural events. The thermocouple signals were acquired similarly along with behavior,

847 but using Synapse software with a sampling rate of $610 \mathrm{~Hz}$.

849 Local field potential analysis

850 To minimize potential multisensory influences (Gnaedinger et al. 2019), all trials analyzed

851 were tone-off trials (Fig. 3B)(Carlson et al. 2018), and came from blocks where behavioral

852 performance was $\geq 80 \%$ correct. For odor only, tone attention, and odor attention, only

853 correct trials were included in all analysis (unless otherwise specified; Fig. 5D). For switch

854 blocks, where the rat is by-definition performing poorly and responding to negative reward

855 feedback, we included correct and incorrect trials. Because only tone-off trials were 856 analyzed, there were twice as many odor only trials compared to tone attention and odor 
857 attention. Therefore, we randomly discarded half of the odor only trials, preserving the

858 proportion that were from the beginning/end of the session as well as the proportion of

859 trial types. The data were imported into MATLAB and traces spanning -10 to 8.4 sec from

860 odor onset from each trial were downsampled to $1 \mathrm{kHz}$, filtered $0.5-100 \mathrm{~Hz}$ using a $2^{\text {nd }}$

861 order bandpass filter, and $59-61 \mathrm{~Hz}$ using a $2^{\text {nd }}$ order band-stop filter. These large

862 segments of data were further filtered to avoid edge artifacts from filtering, but smaller

863 segments were used for later analysis. Power and coherence were computed using the

864 Chronux toolbox (Mitra and Bokil 2009)(http://chronux.org), and raw values were

865 normalized to odor only trials, to identify effects specifically related to attentional demand.

866 Specifically, multi-taper power spectra and coherence were computed using 5 tapers, and

867 mean power was determined by averaging within the given frequency range. For power

868 and PAC analyses, a single LFP trace from the bipolar electrode was used. For coherence

869 analyses, a subtracted trade was first created from the bipolar electrode. For power and

870 coherence analyses, theta was defined as $2-12 \mathrm{~Hz}$, beta as $15-35 \mathrm{~Hz}$, low gamma as $40-$

$87160 \mathrm{~Hz}$, and high gamma as $60-80 \mathrm{~Hz}$.

872 PAC analysis was completed using MATLAB routines from Tort et al., 2010,

873 wherein the Hilbert transform method was used to determine the phase of the carrier

874 oscillation (theta), and separately, the envelope of the high frequency oscillation (beta or

875 high gamma). The amplitude of the beta/high gamma fast oscillation across 51 bins of the

876 theta phase was plotted to demonstrate PAC strength (Fig. 5B). Theta was defined as

$8772-10 \mathrm{~Hz}$, beta defined as $15-35 \mathrm{~Hz}$, and high gamma defined as 65-100 Hz. For each trial,

878 a single OB LFP trace spanning from -3 to $+1.5 \mathrm{sec}$ from odor onset was used. A larger

879 segment of time was used for these analyses because more samples were required to 
880 examine coupling with theta frequencies down to $2 \mathrm{~Hz}$. Still, this time segment allows for

881 the analysis to be contained to an individual trial without any overlap with neighboring

882 trials.

883

Sniffing analysis

885 As with the LFP analyses, all analyses on the sniffing data were restricted to trials where 886 the tone was off. Additionally, only correct trials were examined for odor only, tone 887 attention, and odor attention blocks, while correct and incorrect trials were examined for 888 switch blocks. The data were imported to MATLAB and traces spanning -10 to 8.4 sec 889 from odor onset from each trial were filtered using a $2^{\text {nd }}$ order band-pass filter from 0.5$89010 \mathrm{~Hz}$. After extracting trials as described above for the LFP analyses, these filtered traces

891 were convolved with an $8 \mathrm{~Hz}$ Morlet wavelet and peaks detected. Detected peaks were 892 visually inspected and false positives were manually rejected using a custom MATLAB 893 GUI. The resultant peaks were used to calculate the instantaneous sniffing frequency 894 throughout the trial. Then, instantaneous frequencies were averaged within each trial 895 epoch (i.e. hold, odor), and normalized to odor only.

\section{General statistical methods}

898 Semi-automated routines were used to ensure rigorous data extraction and analyses.

899 Details regarding specific statistical tests can be found in their respective results sections 900 and/or figure legends. Unless otherwise stated, all values are mean \pm SEM. 


\section{References}

904 Adrian E. D. (1942) Olfactory reactions in the brain of the hedgehog. J. Physiol. 100, 459-473.

906

907

908

909

910

911

912

913

914

915

916

917

918

919

920

921

922

923

924

925

Arabkheradmand G., Zhou G., Noto T., Yang Q., Schuele S. U., Parvizi J., Gottfried J. A., et al. (2020) Anticipation-induced delta phase reset improves human olfactory perception. PLoS Biol. 18, e3000724.

Araujo I. E. de, Rolls E. T., Kringelbach M. L., McGlone F., Phillips N. (2003) Tasteolfactory convergence, and the representation of the pleasantness of flavour, in the human brain. Eur J Neurosci 18, 2059-2068.

Bagur S., Lefort J. M., Lacroix M. M., Lavilléon G. de, Herry C., Chouvaeff M., Billand C., Geoffroy H., Benchenane K. (2021) Breathing-driven prefrontal oscillations regulate maintenance of conditioned-fear evoked freezing independently of initiation. Nat. Commun. 12, 2605.

Barker J. M., Taylor J. R., Chandler L. J. (2014) A unifying model of the role of the infralimbic cortex in extinction and habits. Learn. Mem. 21, 441-8.

Bauer A.-K. R., Ede F. van, Quinn A. J., Nobre A. C. (2021) Rhythmic modulation of visual perception by continuous rhythmic auditory stimulation. J. Neurosci. $\mathbf{4 1}$, $7065-7075$.

Beshel J., Kopell N., Kay L. M. (2007) Olfactory bulb gamma oscillations are enhanced with task demands. J Neurosci 27, 8358-8365.

Birrell J. M., Brown V. J. (2000) Medial frontal cortex mediates perceptual attentional set shifting in the rat. $J$ Neurosci 20, 4320-4324.

Biskamp J., Bartos M., Sauer J. F. (2017) Organization of prefrontal network activity by 
respiration-related oscillations. Sci. Rep. 7, 45508.

927 Borgers C., Epstein S., Kopell N. J. (2005) Background gamma rhythmicity and 102, 7002-7007.

Bragin A., Jandó G., Nádasdy Z., Hetke J., Wise K., Buzsáki G. (1995) Gamma (40-100 $\mathrm{Hz}$ ) oscillation in the hippocampus of the behaving rat. J. Neurosci. 15, 47-60.

932 Brassai A., Suvanjeiev R.-G., Bán E.-G., Lakatos M. (2015) Role of synaptic and nonsynaptic glutamate receptors in ischaemia induced neurotoxicity. Brain Res.

935 Bressler S. L., Richter C. G. (2015) Interareal oscillatory synchronization in top-down 936 neocortical processing. Curr. Opin. Neurobiol. 31, 62-66.

937 Buonviso N., Amat C., Litaudon P., Roux S., Royet J. P., Farget V., Sicard G. (2003) 938 Rhythm sequence through the olfactory bulb layers during the time window of a respiratory cycle. Eur J Neurosci 17, 1811-1819.

940 Buzsaki G. (2006) Rhythms of the Brain. Oxford Press.

941 Buzsáki G., Wang X.-J. (2012) Mechanisms of Gamma Oscillations. Annu. Rev.

$942 \quad$ Neurosci. 35, 203-225.

943 Canolty R. T., Edwards E., Dalal S. S., Soltani M., Nagarajan S. S., Kirsch H. E., Berger

944 M. S., Barbare N. M., Knight R. T. (2006) High gamma power is phase-locked to 945 theta oscillations in human neocortex. Science (80-. ). 313, 1626-1628.

946 Cardin J. A., Carlen M., Meletis K., Knoblich U., Zhang F., Deisseroth K., Tsai L.-H., 947 Moore C. I. (2009) Driving fast-spiking cells induces gamma rhythm and controls 948 sensory responses. Nature 459, 663-667. 
949 Carey R. M., Verhagen J. V, Wesson D. W., Pirez N., Wachowiak M. (2009) Temporal

950 Structure of Receptor Neuron Input to the Olfactory Bulb Imaged in Behaving Rats.

951 J Neurophysiol 101, 1073-1088.

952 Carlson K. S., Dillione M., Wesson D. W. (2014) Odor- and state-dependent olfactory

953 tubercle local field potential dynamics in awake rats. J. Neurophysiol. 111, 2109_

9542123.

955 Carlson K. S., Gadziola M. A., Dauster E. S., Wesson D. W. (2018) Selective Attention

956 Controls Olfactory Decisions and the Neural Encoding of Odors. Curr. Biol. 28,

957 2195-2205.e4.

958 Cenier T., McGann J. P., Tsuno Y., Verhagen J. V, Wachowiak M. (2013) Testing the

959 Sorption Hypothesis in Olfaction: A Limited Role for Sniff Strength in Shaping

960 Primary Odor Representations During Behavior. J. Neurosci. 33, 79-92.

961 Chaudhury D., Escanilla O., Linster C. (2009) Bulbar acetylcholine enhances neural and 962 perceptual odor discrimination. J Neurosci 29, 52-60.

963 Clarke S., Trowill J. A. (1971) Sniffing and motivated behavior in the rat. Physiol Behav 964 6, 49-52.

965 Colgin L. L. (2013) Mechanisms and functions of theta rhythms. Annu. Rev. Neurosci.

$966 \quad 36,295-312$.

967 Corbetta M. (1998) Frontoparietal cortical networks for directing attention and the eye to 968 visual locations: Identical, independent, or overlapping neural systems? Proc. Natl. $969 \quad$ Acad. Sci. U. S. A. 95, 831-838.

970 Courtiol E., Wilson D. A. (2016) Neural Representation of Odor-Guided Behavior in the 971 Rat Olfactory Thalamus. J. Neurosci. 36, 5946-5960. 
972 D'Souza R. D., Vijayaraghavan S. (2014) Paying attention to smell: Cholinergic

973 signaling in the olfactory bulb. Front. Synaptic Neurosci. 6.

974 Devore S., Linster C. (2012) Noradrenergic and cholinergic modulation of olfactory bulb 975 sensory processing. Front. Behav. Neurosci.

976 Devoto P., Flore G., Saba P., Fà M., Gessa G. L. (2005) Stimulation of the locus

977 coeruleus elicits noradrenaline and dopamine release in the medial prefrontal and 978 parietal cortex. J. Neurochem. 92, 368-374.

979 Ding D. C. D., Gabbott P. L. A., Totterdell S. (2001) Differences in the laminar origin of projections from the medial prefrontal cortex to the nucleus accumbens shell and core regions in the rat. Brain Res. 917, 81-89.

982 Eeckman F. H., Freeman W. J. (1990) Correlations between unit firing and EEG in the rat olfactory system. Brain Res 528, 238-244.

984 Erö C., Gewaltig M. O., Keller D., Markram H. (2018) A cell atlas for the mouse brain. Front. Neuroinform. 12.

986 Falco E. De, An L., Sun N., Roebuck A., Greba Q., Lapish C., Howland J. (2019) The 987 Rat Medial Prefrontal Cortex Exhibits Flexible Neural Activity States during the 988 Performance of an Odor Span Task. eNeuro 6.

989 Fiebelkorn I. C., Kastner S. (2019) A rhythmic theory of attention. Trends Cogn. Sci. 23, $990 \quad 87$.

991 Fontanini A., Bower J. M. (2006) Slow-waves in the olfactory system: an olfactory perspective on cortical rhythms. Trends Neurosci. 29, 429-437.

993 Fries P. (2015) Rhythms for Cognition: Communication through Coherence. Neuron 88, $994 \quad 220-235$. 
995 Fries P., Reynolds J. H., Rorie A. E., Desimone R. (2001) Modulation of oscillatory

996 neuronal synchronization by selective visual attention. Science (80-. ). 291, 1560-

$997 \quad 1563$.

998 Gabbott P. L. A., Warner T. A., Jays P. R. L., Salway P., Busby S. J. (2005) Prefrontal

999 cortex in the rat: Projections to subcortical autonomic, motor, and limbic centers. J.

$1000 \quad$ Comp. Neurol. 492, 145-177.

1001 Gill T. M., Sarter M., Givens B. (2000) Sustained visual attention performance-

1002 associated prefrontal neuronal activity: Evidence for cholinergic modulation. $J$.

1003 Neurosci. 20.

1004 Gnaedinger A., Gurden H., Gourévitch B., Martin C. (2019) Multisensory learning

1005 between odor and sound enhances beta oscillations. Sci. Rep. 9, 11236.

1006 Gottfried J. A. (2010) Central mechanisms of odour object perception. Nat Rev Neurosci

$1007 \quad 11,628-641$.

1008 Halabisky B., Strowbridge B. W. (2003) Gamma-frequency excitatory input to granule

1009 cells facilitates dendrodendritic inhibition in the rat olfactory Bulb. J Neurophysiol

$101090,644-654$.

1011 Halassa M. M., Kastner S. (2017) Thalamic functions in distributed cognitive control.

$1012 \quad$ Nat. Neurosci. 20, 1669-1679.

1013 Hardung S., Epple R., Jäckel Z., Eriksson D., Uran C., Senn V., Gibor L., Yizhar O.,

1014 Diester I. (2017) A Functional Gradient in the Rodent Prefrontal Cortex Supports

$1015 \quad$ Behavioral Inhibition. Curr. Biol. 27.

1016 Herman A. M., Ortiz-Guzman J., Kochukov M., Herman I., Quast K. B., Patel J. M., 1017 Tepe B., et al. (2016) A cholinergic basal forebrain feeding circuit modulates 
appetite suppression. Nature 538, 253-256.

1019 Himmelheber A. M., Sarter M., Bruno J. P. (2000) Increases in cortical acetylcholine release during sustained attention performance in rats. Cogn. Brain Res. 9, 31325.

1022 Ikemoto S. (2003) Involvement of the Olfactory Tubercle in Cocaine Reward:

1023 Intracranial Self-Administration Studies. J. Neurosci. 23, 9305-9311.

1024 Ikemoto S., Panksepp J. (1994) The relationship between self-stimulation and sniffing in

1025 rats: does a common brain system mediate these behaviors? Behav Brain Res 61, 143-162.

1027 Izquierdo A. (2017) Functional Heterogeneity within Rat Orbitofrontal Cortex in Reward 1028 Learning and Decision Making. J. Neurosci. 37, 10529-10540.

1029 Jensen O., Colgin L. L. (2007) Cross-frequency coupling between neuronal oscillations. $1030 \quad$ Trends Cogn. Sci. 11, 267-9.

1031 Jordan R., Fukunaga I., Kollo M., Schaefer A. T. (2018) Active Sampling State

1032 Dynamically Enhances Olfactory Bulb Odor Representation. Neuron.

1033 Kay L., Freeman W. (1998) Bidirectional processing in the olfactory-limbic axis during 1034 olfactory behavior. Behav. Neurosci. 112, 541-553.

1035 Kay L. M. (2003) Two species of gamma oscillations in the olfactory bulb: dependence on behavioral state and synaptic interactions. J Integr Neurosci 2, 31-44.

1037 Kay L. M., Beshel J., Brea J., Martin C., Rojas-Líbano D., Kopell N. (2009) Olfactory oscillations: the what, how and what for. Trends Neurosci. 32, 207-214.

1039 Kay L. M., Laurent G. (1999) Odor- and context-dependent modulation of mitral cell 1040 activity in behaving rats. Nat Neurosci 2, 1003-1009. 
1041 Kay L. M., Sherman S. M. (2006) An argument for an olfactory thalamus. Trends Neurosci 30, 47-53.

1043 Kepecs A., Uchida N., Mainen Z. F. (2007) Rapid and precise control of sniffing during 1044 olfactory discrimination in rats. J Neurophysio/ 98, 205-213.

1045 Kim H., Ährlund-Richter S., Wang X., Deisseroth K., Carlén M. (2016) Prefrontal

1046 Parvalbumin Neurons in Control of Attention. Cell 164, 208-218.

1047 Kloet S. F. de, Bruinsma B., Terra H., Heistek T. S., Passchier E. M. J., Berg A. R. van

1048 den, Luchicchi A., Min R., Pattij T., Mansvelder H. D. (2021) Bi-directional

1049 regulation of cognitive control by distinct prefrontal cortical output neurons to thalamus and striatum. Nat. Commun. 12, 1994.

Kopell N., Ermentrout G. B., Whittington M. A., Traub R. D. (2000) Gamma rhythms and beta rhythms have different synchronization properties. Proc. Natl. Acad. Sci. U. S. A. 97, 1867-1872.

Lakatos P., Gross J., Thut G. (2019) A New Unifying Account of the Roles of Neuronal Entrainment. Curr. Biol. 29, R890-R905.

1057 Neuronal Oscillations as a Mechanism of Attentional Selection. Science (80-. ). $1058 \quad 320,110-113$.

1059 Laubach M., Amarante L. M., Swanson T. K., White S. R. (2018) What, if anything, is 1060 rodent prefrontal cortex? eneuro, ENEURO.0315-18.2018.

1061 Lefèvre L., Courtiol E., Garcia S., Thévenet M., Messaoudi B., Buonviso N. (2016) 1062 Significance of sniffing pattern during the acquisition of an olfactory discrimination 1063 task. Behav. Brain Res. 312, 341-54. 
1064 Lepousez G., Lledo P.-M. (2013) Odor Discrimination Requires Proper Olfactory Fast 1065 Oscillations in Awake Mice. Neuron 80, 1010-24.

1066 Ljubojevic V., Luu P., Gill P. R., Beckett L.-A., Takehara-Nishiuchi K., Rosa E. De 1067 (2018) Cholinergic Modulation of Frontoparietal Cortical Network Dynamics 1068 Supporting Supramodal Attention. J. Neurosci. 38, 3988-4005.

1069 Losacco J., Ramirez-Gordillo D., Gilmer J., Restrepo D. (2020a) Learning improves 1070 decoding of odor identity with phase-referenced oscillations in the olfactory bulb. $1071 \quad$ Elife 9, e52583.

1072 Losacco J., Ramirez-Gordillo D., Gilmer J., Restrepo D. (2020b) Learning improves 1073 decoding of odor identity with phase-referenced oscillations in the olfactory bulb.

$1074 \quad$ Elife 9.

1075 Luchicchi A., Mnie-Filali O., Terra H., Bruinsma B., Kloet S. F. de, Obermayer J., 1076 Heistek T. S., et al. (2016) Sustained Attentional States Require Distinct Temporal 1077 Involvement of the Dorsal and Ventral Medial Prefrontal Cortex. Front. Neural $1078 \quad$ Circuits 10, 70.

1079 Mably A. J., Colgin L. L. (2018) Gamma oscillations in cognitive disorders. Curr. Opin. $1080 \quad$ Neurobiol. 52, 182-187.

1081 Macrides F. (1975) Temporal relationships between hippocampal slow waves and 1082 exploratory sniffing in hamsters. Behav Biol 14, 295-308.

1083 Macrides F., Eichenbaum H. B., Forbes W. B. (1982) Temporal relationship between $1084 \quad$ sniffing and the limbic theta rhythm during odor discrimination reversal learning. $J$ $1085 \quad$ Neurosci 2, 1705-1711.

1086 Marquis J.-P., Killcross S., Haddon J. E. (2007) Inactivation of the prelimbic, but not 
infralimbic, prefrontal cortex impairs the contextual control of response conflict in rats. Eur. J. Neurosci. 25, 559-566.

Martin C., Ravel N. (2014) Beta and gamma oscillatory activities associated with olfactory memory tasks: Different rhythms for different functional networks? Front. Behav. Neurosci. 8.

McAlonan K., Cavanaugh J., Wurtz R. H. (2008) Guarding the gateway to cortex with attention in visual thalamus. Nature 456, 391-394.

1094 McCormick D. A., Feeser H. R. (1990) Functional implications of burst firing and single spike activity in lateral geniculate relay neurons. Neuroscience 39, 103-113. Chemoanatomical organization of the noradrenergic input from locus coeruleus to the olfactory bulb of the adult rat. J Comp Neurol 285, 339-349.

1099 Merre P. Le, Ährlund-Richter S., Carlén M. (2021) The mouse prefrontal cortex: Unity in diversity. Neuron 109, 1925-1944.

1101 Miller E. K., Cohen J. D. (2001) An Integrative Theory of Prefrontal Cortex Function.

$1102 \quad$ Annu. Rev. Neurosci. 24, 167-202.

1103 Mitra P., Bokil H. (2009) Observed Brain Dynamics.

1104 Miura K., Mainen Z. F., Uchida N. (2012) Odor Representations in Olfactory Cortex:

1105 Distributed Rate Coding and Decorrelated Population Activity. Neuron 74, 1087$1106 \quad 1098$.

1107 Moberly A. H., Schreck M., Bhattarai J. P., Zweifel L. S., Luo W., Ma M. (2018)

1108 Olfactory inputs modulate respiration-related rhythmic activity in the prefrontal $1109 \quad$ cortex and freezing behavior. Nat. Commun. 9, 1528. 
1110 Murata K., Kanno M., leki N., Mori K., Yamaguchi M. (2015) Mapping of Learned Odor-

1111 Induced Motivated Behaviors in the Mouse Olfactory Tubercle. J. Neurosci. 35 ,

1112 10581-10599.

1113 Nakayama H., Ibañez-Tallon I., Heintz N. (2018) Cell-Type-Specific Contributions of

1114 Medial Prefrontal Neurons to Flexible Behaviors. J. Neurosci. 38, 4490 LP - 4504.

1115 Neville K. R., Haberly L. B. (2003) Beta and gamma oscillations in the olfactory system

1116 of the urethane-anesthetized rat. J Neurophysio/ 90, 3921-3930.

1117 O'Connor D., Fukui M., Pinsk M., Kastner S. (2002) Attention modulates responses in

1118 the human lateral geniculate nucleus. Nat. Neurosci. 5, 1203-1209.

1119 Ogg M. C., Ross J. M., Bendahmane M., Fletcher M. L. (2018) Olfactory bulb

1120 acetylcholine release dishabituates odor responses and reinstates odor

1121 investigation. Nat. Commun. 9, 1868.

1122 Osinski B. L., Kim A., Xiao W., Mehta N. M., Kay L. M. (2018) Pharmacological

1123 manipulation of the olfactory bulb modulates beta oscillations: Testing model

1124 predictions. J. Neurophysiol. 120.

1125 Passetti F., Dalley J. W., O'connell M. T., Everitt B. J., Robbins T. W. (2000) Increased

1126 acetylcholine release in the rat medial prefrontal cortex during performance of a

1127 visual attentional task. Eur. J. Neurosci. 12.

1128 Paxinos G., Watson C. (1997) The Rat Brain in Stereotaxic Coordinates. Academic

$1129 \quad$ Press.

1130 Perl O., Ravia A., Rubinson M., Eisen A., Soroka T., Mor N., Secundo L., Sobel N.

1131 (2019) Human non-olfactory cognition phase-locked with inhalation. Nat. Hum.

1132 Behav. 201935 3, 501-512. 
1133 Plailly J., d'Amato T., Saoud M., Royet J. P. (2006) Left temporo-limbic and orbital 1134 dysfunction in schizophrenia during odor familiarity and hedonicity judgments.

$1135 \quad$ Neuroimage 29, 302-313.

1136 Plailly J., Howard J. D., Gitelman D. R., Gottfried J. A. (2008) Attention to odor modulates thalamocortical connectivity in the human brain. J Neurosci 28, 5257-

Posner M. I. (2016) ORIENTING OF ATTENTION: THEN AND NOW. Q. J. Exp. 5267.

Posner M. I., Snyder C. R., Davidson B. J. (1980) Attention and the detection of signals. J. Exp. Psychol. Gen. 109.

1143 Ragozzino M. E., Kim J., Hassert D., Minniti N., Kiang C. (2003) The contribution of the rat prelimbic-infralimbic areas to different forms of task switching. Behav. Neurosci.

1145 Richter C. G., Thompson W. H., Bosman C. A., Fries P. (2017) Top-down beta enhances bottom-up gamma. J. Neurosci. 37 .

1147 Rikhye R. V., Gilra A., Halassa M. M. (2018) Thalamic regulation of switching between $1148 \quad$ cortical representations enables cognitive flexibility. Nat. Neurosci. 21.

1149 Rizzolatti G., Riggio L., Dascola I., Umiltá C. (1987) Reorienting attention across the 1150 horizontal and vertical meridians: Evidence in favor of a premotor theory of attention. Neuropsychologia 25.

1152 Rodgers C. C., DeWeese M. R. (2014) Neural Correlates of Task Switching in 1153 Prefrontal Cortex and Primary Auditory Cortex in a Novel Stimulus Selection Task 1154 for Rodents. Neuron 82, 1157-1170.

1155 Rojas-Líbano D., Kay L. M. (2012) Interplay between Sniffing and Odorant Sorptive 
Properties in the Rat. J. Neurosci. 32, 15577-15589.

1157 Rolls E. T. (2004) The functions of the orbitofrontal cortex. Brain Cogn 55, 11-29.

1158 Rothermel M., Carey R. M., Puche A., Shipley M. T., Wachowiak M. (2014) Cholinergic

1159 Inputs from Basal Forebrain Add an Excitatory Bias to Odor Coding in the Olfactory

$1160 \quad$ Bulb. J. Neurosci. 34, 4654-4664.

1161 Sacchet M. D., LaPlante R. A., Wan Q., Pritchett D. L., Lee A. K. C., Hämäläinen M.,

1162 Moore C. I., Kerr C. E., Jones S. R. (2015) Attention drives synchronization of

1163 alpha and beta rhythms between right inferior frontal and primary sensory

1164 neocortex. J. Neurosci. 35.

1165 Samuelsen C. L., Gardner M. P. H., Fontanini A. (2013) Thalamic Contribution to

1166 Cortical Processing of Taste and Expectation. J. Neurosci. 33, 1815-1827.

1167 Santana N., Artigas F. (2017) Laminar and Cellular Distribution of Monoamine

1168 Receptors in Rat Medial Prefrontal Cortex. Front. Neuroanat. 11, 87.

1169 Sara S. J. (2009) The locus coeruleus and noradrenergic modulation of cognition.

1170 Schmitt L. I., Wimmer R. D., Nakajima M., Happ M., Mofakham S., Halassa M. M.

1171 (2017) Thalamic amplification of cortical connectivity sustains attentional control.

$1172 \quad$ Nature 545, 219-223.

1173 Schoenbaum G., Roesch M. R., Stalnaker T. A., Takahashi Y. K. (2009) A new

1174 perspective on the role of the orbitofrontal cortex in adaptive behaviour. Nat Rev

$1175 \quad$ Neurosci 10, 885-892.

1176 Schoppa N. E. (2006) Synchronization of olfactory bulb mitral cells by precisely timed

1177 inhibitory inputs. Neuron 49, 271-283.

1178 Schroeder C. E., Lakatos P. (2009a) The Gamma Oscillation: Master or Slave? Brain 
Topogr. 22, 24-26.

1180 Schroeder C. E., Lakatos P. (2009b) Low-frequency neuronal oscillations as instruments of sensory selection. Trends Neurosci. 32, 9-18. responses tile the sniff cycle. Nat Neurosci 14, 1039-1044.

1184 Siegle J. H., Pritchett D. L., Moore C. I. (2014) Gamma-range synchronization of fastspiking interneurons can enhance detection of tactile stimuli. Nat. Neurosci. 17.

1186 Small D. M., Zatorre R. J., Dagher A., Evans A. C., Jones-Gotman M. (2001) Changes in brain activity related to eating chocolate: From pleasure to aversion. Brain 124,

Sobel E. C., Tank D. W. (1993) Timing of odor stimulation does not alter patterning of

Spence C., Kettenmann B., Kobal G., McGlone F. P. (2000) Selective attention to the olfactory bulb unit activity in freely breathing rats. J Neurophysiol 69, 1331-1337.

Spellman T., Svei M., Kaminsky J., Manzano-Nieves G., Liston C. (2021) Prefrontal

1196 Spitzer B., Haegens S. (2017) Beyond the Status Quo: A Role for Beta Oscillations in Endogenous Content (Re)Activation. eneuro 4, ENEURO.0170-17.2017.

1198 Spors H., Wachowiak M., Cohen L. B., Friedrich R. W. (2006) Temporal dynamics and latency patterns of receptor neuron input to the olfactory bulb. J Neurosci 26, 1247$1200 \quad 1259$.

1201 Thompson K. G., Biscoe K. L., Sato T. R. (2005) Neuronal basis of covert spatial 
attention in the frontal eye field. J. Neurosci. 25.

1203 Tort A. B. L., Brankačk J., Draguhn A. (2018a) Respiration-Entrained Brain Rhythms

$1204 \quad$ Are Global but Often Overlooked. Trends Neurosci.

1205 Tort A. B. L., Ponsel S., Jessberger J., Yanovsky Y., Brankačk J., Draguhn A. (2018b)

1206 Parallel detection of theta and respiration-coupled oscillations throughout the

1207 mouse brain. Sci. Rep. 8.

1208 Tort A., Komorowski R., Eichenbaum H., Kopell N. (2010) Measuring phase-amplitude

1209 coupling between neuronal oscillations of different frequencies. J. Neurophysiol.

$1210 \quad 104,1195-1210$.

1211 Uchida N., Kepecs A., Mainen Z. F. (2006) Seeing at a glance, smelling in a whiff: rapid 1212 forms of perceptual decision making. Nat Rev Neurosci 7, 485-491.

1213 Uchida N., Mainen Z. F. (2003) Speed and accuracy of olfactory discrimination in the

1214 rat. Nat Neurosci 6, 1224-1229.

1215 Vanderwolf C. H. (1992) Hippocampal activity, olfaction, and sniffing: an olfactory input

1216 to the dentate gyrus. Brain Res. 593, 197-208.

1217 VanRullen R. (2016) Perceptual Cycles. Trends Cogn. Sci. 20, 723-735.

1218 Verhagen J. V, Wesson D. W., Netoff T. I., White J. A., Wachowiak M. (2007) Sniffing 1219 controls an adaptive filter of sensory input to the olfactory bulb. Nat Neurosci 10, $1220 \quad 631-639$.

1221 Vertes R. P. (2004) Differential projections of the infralimbic and prelimbic cortex in the $1222 \quad$ rat. Synapse 51, 32-58.

1223 Vinck M., Womelsdorf T., Buffalo E. A., Desimone R., Fries P. (2013) Attentional 1224 Modulation of Cell-Class-Specific Gamma-Band Synchronization in Awake Monkey 
Area V4. Neuron 80, 1077-1089.

Wang P., Boboila C., Chin M., Higashi-Howard A., Shamash P., Wu Z., Stein N., Abbott Prefrontal Cortex. Neuron 108, 209-224.e6.

Wang X. J. (2010) Neurophysiological and computational principles of cortical rhythms in cognition.

1231 Welker W. I. (1964) Analysis of sniffing in the albino rat. Behavior 22, 223-244.

1232 Wesson D. W. (2013) Sniffing Behavior Communicates Social Hierarchy. Curr. Biol. 23, $575-580$.

1234 Wesson D. W. (2020) The Tubular Striatum. J. Neurosci. 40, 7379-7386.

1235 Wesson D. W., Donahou T. N., Johnson M. O., Wachowiak M. (2008) Sniffing behavior 1236 of mice during performance in odor-guided tasks. Chem Senses 33, 581-596.

1237 Wesson D. W., Verhagen J. V, Wachowiak M. (2009) Why Sniff Fast? The Relationship 1238 Between Sniff Frequency, Odor Discrimination, and Receptor Neuron Activation in 1239 the Rat. J Neurophysiol 101, 1089-1102.

1240 Wimmer R. D., Schmitt L. I., Davidson T. J., Nakajima M., Deisseroth K., Halassa M. M. 1241 (2015) Thalamic control of sensory selection in divided attention. Nature 526, 7051242709.

1243 Youngentob S. L., Mozell M. M., Sheehe P. R., Hornung D. E. (1987) A quantitative 1244 analysis of sniffing strategies in rats performing odor discrimination tasks. Physiol 1245 Behav 41, 59-69.

1246 Yu A. J., Dayan P. (2005) Uncertainty, Neuromodulation, and Attention. Neuron 46, $1247 \quad 681-692$. 
1248 Zaborszky L., Carlsen J., Brashear H. R., Heimer L. (1986) Cholinergic and GABAergic

1249 afferents to the olfactory bulb in the rat with special emphasis on the projection

1250 neurons in the nucleus of the horizontal limb of the diagonal band. J Comp Neurol

$1251 \quad 243,488-509$.

1252 Zelano C., Bensafi M., Porter J., Mainland J., Johnson B., Bremner E., Telles C., Khan

1253 R., Sobel N. (2005) Attentional modulation in human primary olfactory cortex. Nat

$1254 \quad$ Neurosci 8, 114-120.

1255 Zhang Z., Collins D. C., Maier J. X. (2021) Network Dynamics in the Developing Piriform

$1256 \quad$ Cortex of Unanesthetized Rats. Cereb. Cortex 31.

1257 Zhang Z., Liu Q., Wen P., Zhang J., Rao X., Zhou Z., Zhang H., et al. (2017) Activation

1258 of the dopaminergic pathway from VTA to the medial olfactory tubercle generates

1259 odor-preference and reward. Elife 6, e25423.

1260 Zhong W., Ciatipis M., Wolfenstetter T., Jessberger J., Müller C., Ponsel S., Yanovsky

1261 Y., Brankačk J., Tort A. B. L., Draguhn A. (2017) Selective entrainment of gamma

1262 subbands by different slow network oscillations. Proc. Natl. Acad. Sci. U. S. A. 114.

1263

1264 
A

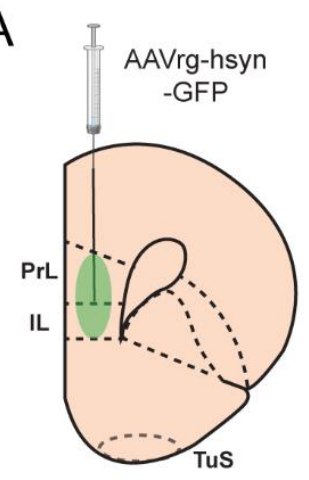

B

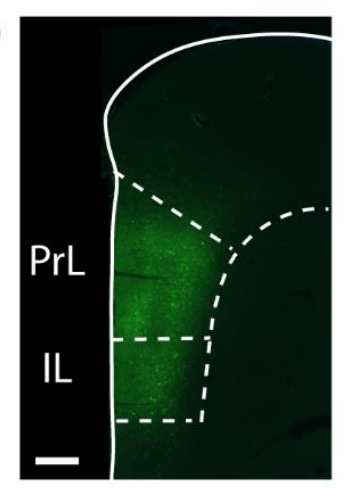

C

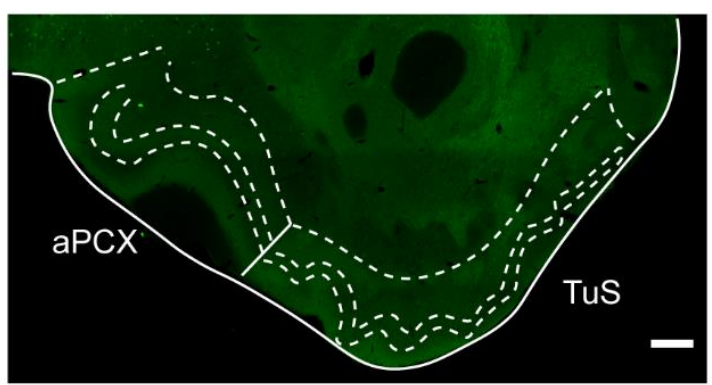

Supplemental Figure S1. PCX and TuS projection neurons do not innervate the mPFC. A. The PrL and IL were injected with AAVrg-hsyn-GFP to identify possible mPFC-projecting neurons. B. Example injection site showing spread through the PrL and IL cortex. Scale bar $250 \mu \mathrm{m}$. C. Example image showing lack of labeled cells in the TuS and APCX, indicating that these structures do not project to the mPFC. Similar results were seen in 2 rats. Scale bar $250 \mu \mathrm{m}$. 
A

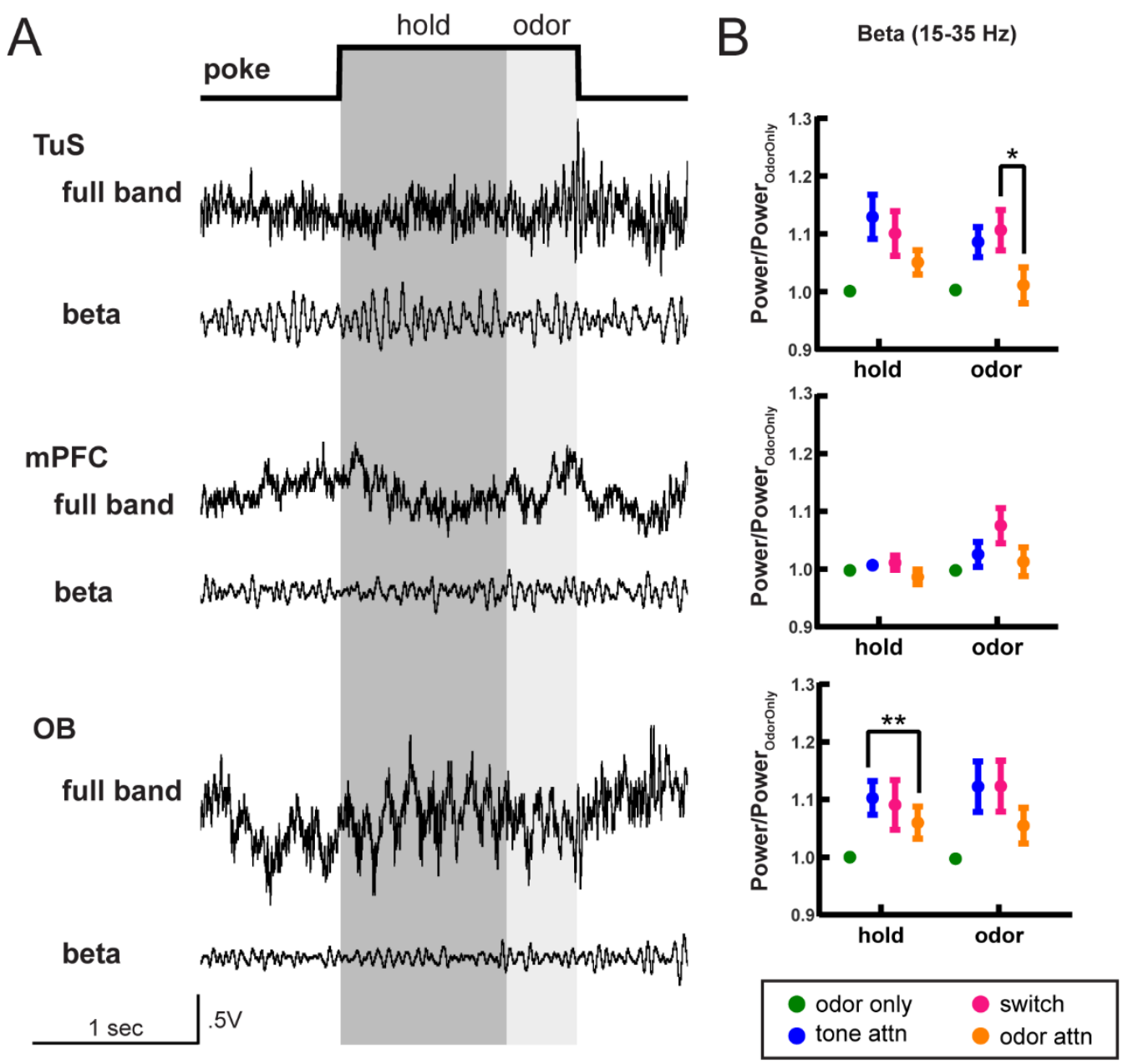

B Beta $(15-35 \mathrm{~Hz})$

Supplemental Figure S2. Beta oscillation power during attentional states. A. Full band and beta band filtered $(15-35 \mathrm{~Hz}$ ) traces from the TuS (top), mPFC (middle) and OB (bottom) on a single trial of the Carlson Attention task. Analysis windows for hold and odor periods are indicated in dark and light gray, respectively. B. Quantification of power in the beta range across all task types, normalized to odor only. Statistical tests were 2-way ANOVA with Geisser-Greenhouse correction. TuS: main effect of task type, $F(1.94,7.77)=16.37, p=0.0017$. mPFC: main effect of task type, $F(2.00,8.02)=4.923, p=0.0402$. OB: main effect of task type, $F(1.49,5.97)=16.52$, $p=0.0047$. On all graphs, asterisks indicate results from Tukey's multiple comparisons, ${ }^{*} p<0.05$, ${ }^{* *} p<0.01$. All error bars represent SEM. $n=5$ rats, $4.6+/-0.5$ sessions per rat. 

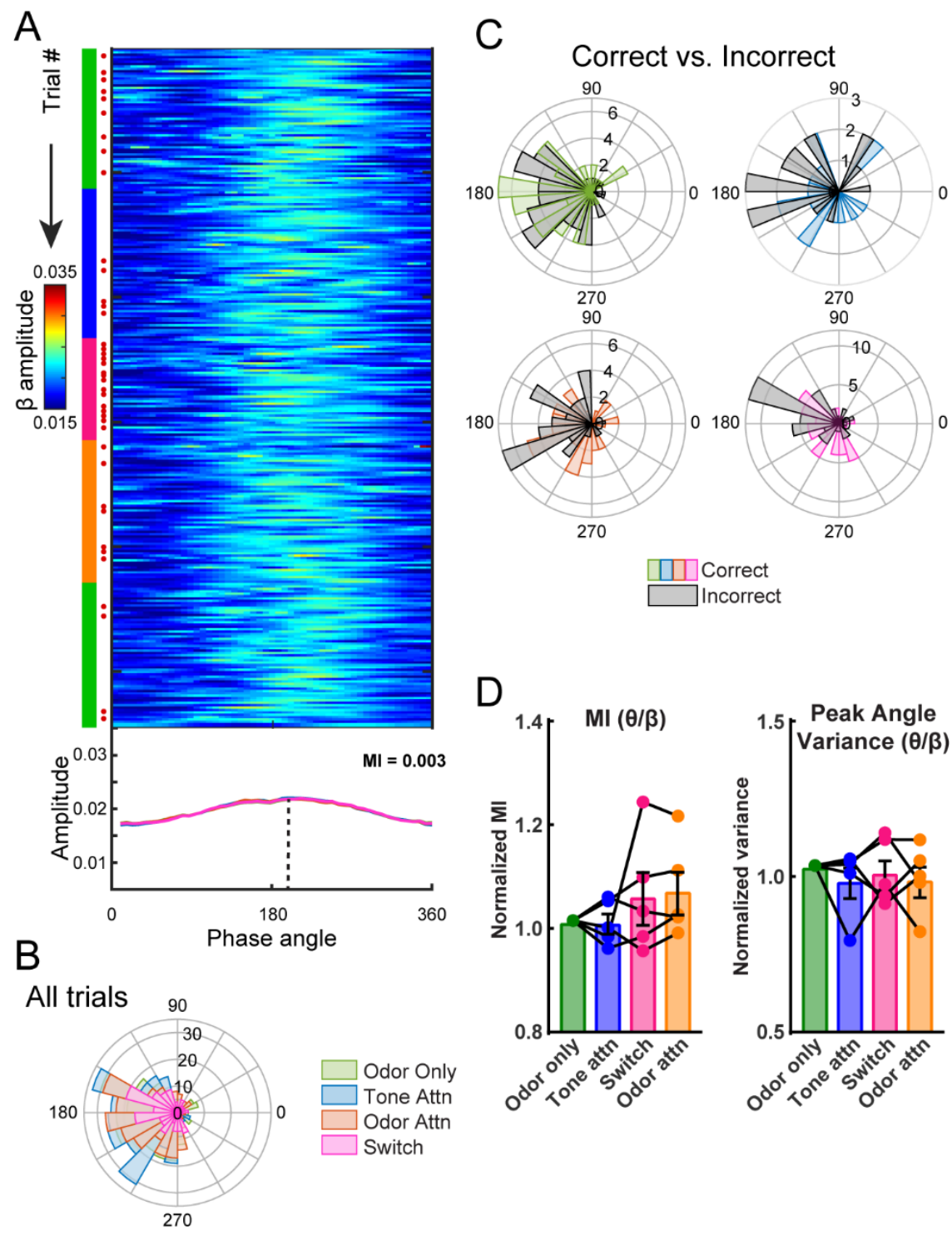

Supplemental Figure S3. Modest theta-beta phase amplitude coupling in the CAT. A. Trial by trial theta-beta PAC for one example session. Green, blue, pink, and orange markings on the left side indicate current task type (odor only, tone attention, switch, and odor attention, respectively). Red dots indicate incorrect trials. The mean amplitude for the session, by task type, is plotted below. MI for the entire session $=0.003$. B. Polar histogram of peak phase angles by task type for all sessions for this example rat ( $n=5$ sessions). All trial types showed significant periodicity (Rayleigh test, odor only $p<1 \mathrm{e}-27$, tone attn $\mathrm{p}<1 \mathrm{e}-8$, switch $\mathrm{p}<0.05$, odor attn $\mathrm{p}<1 \mathrm{e}-12$ ) and similar distributions (Kolmogorov-Smirnov tests, $p>0.05$ for all comparisons except odor only vs. switch, $p=0.046)$. C. Polar histograms showing correct and incorrect trials for each type. For this example rat, incorrect trials were pooled across sessions and compared to a randomly selected equal number of correct trials ( $n=5$ sessions). Peak phase angle distributions were statistically similar between correct and incorrect trials for odor only, odor attention, and switch trials (Kolmogorov-Smirnov tests, $p>0.05$ ) but statistically different for tone attention $(p=0.041)$. D. Left, theta-beta $\mathrm{Ml}$ across rats, normalized to $\mathrm{Ml}$ for odor only trials (One-way ANOVA, $F(1.19,4.78)=1.22, p=0.34)$. Right, theta-beta peak angle variance across rats, normalized to peak angle variance for odor only trials (One-way ANOVA, $F(2.18,8.31)=0.35, p=0.72) . n=5$ rats, 4.6 $+/-0.5$ sessions per rat. 